\title{
Duals of Crystallographic Groups. Band and Quasi-Band Representations
}

\author{
Henri Bacry ${ }^{\star}$ \\ Department of Physics, Technion-Israel Institute of Technology, Haifa 32000, Israel
}

Received May 27, 1992; in revised form July 10, 1992

\begin{abstract}
Band representations are analyzed from a pure group theoretical point of view, with the aid of the dual of the crystallographic group (the set of equivalence classes of unitary irreducible representations). It is shown on the examples of the onedimensional crystallographic groups that we have to introduce a distinction between band and quasi-band representations, the word band being reserved for induced representations.

The dual of the group $F 222$ is explicitly constructed. It permits to show that two elementary band representations which have the same decompositions into unitary irreducible representations are not equivalent.
\end{abstract}

\section{Introduction}

Generally, in physics, the crystallographic groups (also called space groups) are studied not as abstract groups but from their Euclidean properties, that is from crystals. However, many of their properties can be investigated from a strictly group theoretical point of view. Here, we propose to present some interesting facts concerning the orthorhombic face centered group $F 222$ (number 22 in the International Tables for Crystallography [1]). The main parts of the work which is presented here are i) the construction of the dual, that is the space of all classes of irreducible representations of the group and ii) the analysis of some band representations [2-12]. In many respects, such an approach is different from the one based on the Brillouin zone and Hamiltonian theory. From the point of view of representation theory, the Brillouin zone is an object which provides a nice way of constructing the unitary irreducible representations of the space group but, although it is related with the open part of the dual, it hides all properties of band representations which are essentially related with the topological structure of the dual. Another inconvenience of the Brillouin

\footnotetext{
* On leave from Centre de Physique Théorique and Faculté des Sciences de Luminy, Case 907, 13288 Marseille, Cedex 8, France
} 
zone occurs for non-symmorphic groups for which the dual is a kind of a covering of the Brillouin zone, playing the role of a Riemann sheet with respect to it. Even in very simple groups as $F 222$, when we are dealing with band representations, the dual permits to discover properties which cannot be seen easily in the Brillouin zone approach. Let us explain why.

If we except the Euclidean group itself and its one and two-dimensional Euclidean subgroups, all the subgroups of this group which are used in physics have a property in common: all their unitary irreducible representations (unirreps) are finite dimensional. These groups belong to one of the following categories:

1) The compact Lie subgroups,

2) the finite subgroups of $\mathrm{O}(3)$,

3) the crystallographic subgroups (space subgroups).

The dual of these groups have properties which depend on the category it belongs to. The dual of an Abelian group is known to be itself an Abelian group. Moreover, if this group is finite, the dual has as many elements as the group itself. At first sight, topology seems to be involved in the compact Lie subgroups only. This is wrong. In a sense, one even can say that a crystallographic group is the symmetric of a compact Lie group. A compact Lie group has obvious topological properties but its dual, that is its set of classes of unitary irreducible representations is discrete. On the contrary, a crystallographic group is discrete but its dual is continuous and therefore has topological properties which must be examined as such. These properties are more complex than the ones of Lie groups since, except in some trivial cases, the dual of a crystallographic group is quasi compact, i.e. it satisfies compactness properties except that is not Hausdorff (not separated) [13]. Compact Lie groups and space groups have a property in common: their unirreps are finite dimensional; we note that the unirreps of a crystallographic group are of bounded dimension.

The crystallographic groups, the finite groups, the compact Lie groups, the semisimple Lie groups belong to a class of groups which have an interesting property: the reduction of any representation into unirreps is unique. Almost all the subgroups of the Euclidean subgroup we have listed above share also the following property: two representations are equivalent if and only if they have the same content in unirreps. Although we have to give this sentence a more precise meaning, we can already say that there are infinite dimensional representations of crystallographic groups for which non-equivalent representations may have the same content in irreducible representations. In particular, there are 3-dimensional crystallographic groups for which non-equivalent band representations have the same decomposition in unirreps. One of these groups is the cubic group $F 222$. This is the reason why we decided to build explicitly the dual of this group.

The existence of topological properties in the case of a crystallographic crystal has another important consequence: when one replaces a crystallographic group $G$ by one of its corresponding Born-von Karman analog, one must acknowledge a loss of information, just because the Born-von Karman groups are finite. We note that if these groups do not appear in our list, it is because they are not subgroups of the Euclidean group. They are rather quotient groups of crystallographic groups as the group $\mathrm{SO}(3)$ is a quotient group of $S U(2)$. The dual of a quotient group is just a subset of the dual of a group. No simple property of that kind relates the dual of a subgroup to the dual of a group! In our particular cases, the dual of a Born-von Karman group is a subset of the dual of the corresponding crystallographic group as the dual of $\mathrm{SO}(3)$, 
isomorphic to the set of natural integers (the spin), is a subset of the dual of SU(2), namely the union of natural integers and half-integers.

It is not only for pedagogical purposes that we decided not to present immediately our investigation on the group $F 222$. We first apply our analysis to the onedimensional crystallographic groups. This will permit us to provide the reader with group theoretical definitions of some concepts which are usually introduced with the aid of Euclidean properties of crystals. The main advantage of this preliminary study is the presentation of interesting facts concerning the general representations of the crystallographic groups, already visible on these elementary examples. We repeat that our study is independent of Hamiltonian theory, although any periodic Hamiltonian can be studied from the point of view of representations theory. Here, the notion of band representation is introduced directly as a group theoretical concept. However, to be more precise, we felt obligated to define as a more general representation, that of a quasi-band representation.

\section{The Trivial One-Dimensional Space Group}

1. Definition. We can define the elements of this group with the aid of the matrices

$$
t^{n}=\left(\begin{array}{ll}
1 & n \\
0 & 1
\end{array}\right)
$$

where $n$ is an integer (positive, negative or zero).

That it is a crystallographic group can be seen from its action on the real line. If $x$ is a real number, one has

$$
t^{n}\left(\begin{array}{l}
x \\
1
\end{array}\right)=\left(\begin{array}{ll}
1 & n \\
0 & 1
\end{array}\right)\left(\begin{array}{l}
x \\
1
\end{array}\right)=\left(\begin{array}{c}
x+n \\
1
\end{array}\right) .
$$

The definition (2.1) provides us with a finite dimensional representation of the group. This is, by definition, a faithful representation. We will see that all finite unitary representations of $G$ by rational matrices are unfaithful. This does not contradict the fact that the two-dimensional representation (2.1) is rational since it is not a unitary representation.

2. Unitary Irreducible Representations (Unirreps). In the present article, the word representation is used with its standard meaning, namely the one of "class of equivalent representations."

Since the element $t$ is a generator, we only have to know the operator or the matrix which represents it to define a representation. The group being Abelian, all it unirreps are one-dimensional. If $k$ denotes an angle $(-\pi<k \leq \pi)$, it labels the unirrep defined by $t=\exp (-i k)$. It readily follows that the dual of the group, that is the set of all unirreps, has the topology of a circle. We will refer to this circle as the Brillouin torus. This denomination is justified in the following way: when $k$ is considered as a real variable running from $-\infty$ to $+\infty$, it is called the quasi-momentum. Each "zone" of the form $2 \pi\left(n-\frac{1}{2}\right)<k \leq 2 \pi\left(n+\frac{1}{2}\right)$ is known as a Brillouin zone.

It is known that the dual of an Abelian group can be given a group structure, the unit element of it being the trivial representation. Here the dual group is isomorphic to $U(1)$, the group law of the dual is given by $\exp (-i k) \exp \left(-i k^{\prime}\right)=\exp \left(-i k-i k^{\prime}\right)$ and the trivial representation corresponds to the value $k=0$. We note that the dual 
of the dual is isomorphic to the group itself, the representations of $U(1)$ are labelled by an integer (usually denoted by physicists by $m$, corresponding to $t^{m}$ ).

3. The Regular Representation. By definition, the regular representation is the one defined by the action of the group on the space of functions on the group itself. To be more precise, let us introduce a basis for this space. The basis functions we have in mind are $e_{n}$ ( $n$ going from $-\infty$ to $+\infty$ ) where $e_{n}\left(n^{\prime}\right)=\delta_{n n^{\prime}}$. That it is a basis is obvious since any function $f$ can be written as a linear combination

$$
f=f_{0} e_{0}+\sum_{n=1}^{\infty}\left(f_{n} e_{n}+f_{-n} e_{-n}\right) .
$$

The space we are dealing with is more usually supposed to be made of all functions such that

$$
\left|f_{0}\right|^{2}+\sum_{n=1}^{\infty}\left(\left|f_{n}\right|^{2}+\left|f_{-n}\right|^{2}\right)<\infty .
$$

It is the Hilbert space named $l^{2}$; its name comes from the hypothesis that the basis we have defined is an orthonormal basis.

It is clear that the generator $t$ acts as follows:

$$
t^{(\mathrm{reg})} e_{n}=e_{n+1} \text {. }
$$

4. The Band Representation. Quasi-Band Representations. Let us consider a square integrable function $f(x)$. We can make each element of the group acting on that function. The representation we obtain in this way is described by the action of the generator $t$. The corresponding operator $T$ acts as follows:

$$
(T f)(x)=f(x-1), \quad\left(T^{n} f\right)(x)=f(x-n)=f_{n}(x), \quad\left(T f_{n}\right)(x)=f_{n+1}(x) .
$$

The functions $f_{n}(x)$ span a space which is the representation space. We decide to call such a representation a quasi-band representation. It is a unitary representation since the group maps the square integrable function $f$ on square integrable functions. Whenever these functions are linearly independent (which is obviously the case, for instance, when the function $f$ has a support with length less than 1), the representation obtained does not depend on the function $f$. In that case it is the representation induced by the trivial representation (the group is trivially acting on the one-dimensional space which the function $f$ belongs to). It is the (unique) band representation of the group; since the $f_{n^{\prime}} s$ form a basis of the representation space, this representation is easily shown to be equivalent to the regular representation. According to the meaning of the word representation we have adopted, the band representation is the regular representation.

In the present work, we must underline that the words "band" and "quasi-band" have a pure group theoretical significance. This does not mean that we are far from physics. The problem we are studying here is physical. Any square integrable function $f$ describes a state $|f\rangle$. Any Euclidean transformed of $f$ describes also a state. Consider any subgroup $G$ of the Euclidean group. When it acts on $f$ it gives rise to a Hilbert space. Such a space describes a subset of states for a spinless particle. The decomposition of the representation into irreducible representations of the group $G$ is a problem which is well stated [14]. If the particle one is studying is lying in a periodic structure, this structure involves a symmetry group (a crystallographic group). It is 
natural to know how to decompose a quasi-band representation of a crystallographic group. The only point is that no reference to periodic potential theory is needed to state that the space we are interested in has a physical meaning. The symmetry point of view is the only one which is used in the present analysis.

When a Hamiltonian with a periodical potential is introduced, assumptions are usually made about this potential from which band theory is derived. One of the accepted results of that theory is that the energy spectrum is composed of bands and that, for a given band, the energy is a function defined everywhere on the Brillouin zone. The definition we proposed in the present article of the word "band representation" is compatible with this assumption and this is a justification for such an expression. However, from a pure quantum point of view, a quasi-band representation is as physical as a band representation.

5. Decomposition on the Regular Representation into Irreducible Representations. The problem of reducing a given unitary representation $D$ of a group $G$ on a Hilbert space $H$ is a well stated problem from the point of view of the physicist. When $G$ is compact or finite, we know that $H$ can be written as the direct sum of Hilbert subspaces on which the group acts irreducibly and unitarily.

Whenever the group is infinitely discrete as it is the case when we deal with a crystallographic group, the problem is more subtle [14], essentially because the decomposition of a representation does not coincide with the search of invariant subspaces. In order to illustrate that point, we examine the case of the trivial one-dimensional crystallographic group. The problem is to know how many times the representation $D^{(k)}$ is contained in the representation $D$. A given unitary representation $D$ can be decomposed in a combination of direct integrals and direct sums in the following way:

$$
D=\int_{k_{1}}^{k_{2}} D^{(k)} d k+\int_{k_{3}}^{k_{4}} D^{(k)} d k+\cdots+D^{(m)}+D^{(n)}+\cdots
$$

The corresponding spectral decomposition of the unit operator $I$ on the space $H$ is

$$
I=\int_{k_{1}}^{k_{2}} P^{(k)} d k+\int_{k_{3}}^{k_{4}} P^{(k)} d k+\cdots+P^{(m)}+P^{(n)}+\cdots
$$

where $P^{(k)} d k$ denotes a continuous projection-valued measure and the $P^{(m)}$ denote self-adjoint projections, all of them of rank one (the dimensionality of irreducible representations).

Equation (2.2a) gives the content of $D$ in unirreps. When $D^{(k)}$ appears in the direct sum part of $D$ decomposition, it is a subrepresentation of $D$. It means that there exists a Hilbert subspace $H^{(k)}$ of $H$ on which the group acts irreducibly and unitarily. If $D^{(k)}$ appears in one of the direct integrals, it is not a subrepresentation.

Let us now examine the unirreps contents of the regular representation of the group $G$. It can be shown that each unirrep is contained once in the regular representation. To prove it in the present case, we look for a linear combination of functions $f_{n}(x)$, namely $\psi_{k}(x)$ such that $T \psi_{k}(x)=e^{-\imath k} \psi_{k}(x)$. It is easy to see that the only function 
(up to a factor) is given by the Bloch function

$$
\psi_{k}(x)=\sum_{-\infty}^{+\infty} e^{i n k} f_{n}(x)=\sum_{-\infty}^{+\infty} e^{i n k} f(x-n)
$$

From the uniqueness of this eigenfunction, one deduces that each unirrep is contained once and only once in the regular representation. We have

$$
D^{(\mathrm{reg})}=\int_{B . \text { torus }} D^{(k)} d k=\int_{-\pi}^{+\pi} D^{(k)} d k
$$

Remark. It could happen that $D^{(k)}$ vanishes on a set of measure zero. For instance, the function $\psi_{k}(x)$ may vanish for some value of $k$. This does not change the definition of the integral (2.4). However, a unirrep is missing in the decomposition. We are now going to examine that case with help of the Fourier transform.

6. Fourier Transform. Fourier transform permits to see precisely how the $f_{n^{\prime}} s$ can be linearly dependent. Let us denote by $\hat{f}_{n}(p)$ the Fourier transform of the function $f_{n}(x)$. The action of $T$ on $\hat{f}_{n}(p)$ is given by

$$
T \hat{f}_{n}(p)=\int_{-\infty}^{\infty} T f_{n}(x) e^{-i p x} d x=\int_{-\infty}^{\infty} f_{n}(x-1) e^{-i p x} d x=\int_{-\infty}^{\infty} e^{-i p} f_{n}(x) e^{-i p x} d x
$$

that is

$$
T \hat{f}_{n}(p)=e^{-i p} \hat{f}_{n}(p) \text { with } \hat{f}_{n}(p)=e^{-i p n} \hat{f}(p) .
$$

The Fourier transform of the Bloch function is (in using the Poisson formula)

$$
\begin{aligned}
\widehat{\psi}_{k}(p) & =\sum_{-\infty}^{+\infty} e^{i n k} \hat{f}_{n}(p)=\sum_{-\infty}^{+\infty} e^{i n(k-p)} \hat{f}(p)=2 \pi \sum_{-\infty}^{+\infty} \delta(p-k-2 \pi n) \hat{f}(p) \\
& =2 \pi \sum_{-\infty}^{+\infty} \delta(p-k-2 \pi n) \hat{f}(k+2 \pi n) .
\end{aligned}
$$

The action of the generator of that function is described, as expected, by

$$
T \widehat{\psi}_{k}(p)=2 \pi \sum_{-\infty}^{+\infty} e^{-i p} \delta(p-k-2 \pi n) \hat{f}(p)=e^{-\imath k} \widehat{\psi}_{k}(p)
$$

If the function $\widehat{\psi}_{k}(p)$ vanishes at some point $p$, the representation is not induced. For that, $\hat{f}(p)$ must have periodic zeros. It is the case, for instance, if $\hat{f}(p)$ is of the form

$$
\hat{f}(p)=P(\cos p, \sin p) g(p),
$$

where $P$ is a function vanishing for some value of $p$. An example of such a function is given by

$$
f(x)=\exp \left(-\frac{(x+1)^{2}}{2}\right)-\exp \left(-\frac{(x-1)^{2}}{2}\right) \text {. }
$$


The Fourier transform has periodic zeros ${ }^{1}$. It is, up to a factor, the function

$$
\hat{f}(p)=\sin p \exp \left(-\frac{p^{2}}{2}\right) .
$$

We note that the quasi-band representation obtained in this way is not equivalent to the regular representation. Although it has the same decomposition (2.4), we cannot say that each unirrep is contained once (and only once) as it was the case for the regular representation.

7. The Associated Born-von Karman Groups. We just saw that two representations which have the same decomposition may be non-equivalent, a fact which never happens for finite groups. We must underline at this stage that solid state physicists use to impose to the eigenfunctions of the Hamiltonian periodic conditions known as Bornvon Karman conditions. Such conditions can be interpreted as the replacement of the crystallographic group by a finite group often referred to as a Born-von Karman group. The Born-von Karman groups of a space group $G$ of dimension $n$ can be defined as quotient groups of $G$, namely $G / H$, where $H$ is an invariant translation subgroup of the same dimension $n$. When the group $H$ is the entire translation subgroup of $G$, it is called the point group of $G$. The unirreps of a quotient group $G / H$ is a subset of the dual of $G$. In other words, the dual of $G / H$ is a subset of the dual of $G$. Since the Born-von Karman groups are always finite, their duals are always finite subsets of the dual of $G$. In the present case, the Born-von Karman groups are the groups defined by (2.1) with the condition $t^{N}=1$ or, equivalently, with the matrix entries defined modulo $N$. For $N=1$ we get the point group which is the trivial group with one element.

8. Concluding Remark. Let us denote by $G^{*}$ the dual of an arbitrary space group $G$. Among the compact subsets there are ones which are not contained in other compact subsets. We call them the maximal compact subduals. It is clear that in the case we have just studied, the dual $G^{*}$ is the only maximal compact subdual and the only band representation of $G$ has a simple decomposition on it.

\section{The Non-Trivial One-Dimensional Space Group}

There are only two distinct one-dimensional crystallographic groups, the one we just studied (which is referred to sometimes as the infinite cyclical group) and the following one (which is known as the infinite dihedral group).

1. Definition. This group, hereafter denoted by $G$, can be defined as the group of $2 \times 2$ matrices generated by the two following matrices:

$$
t=\left(\begin{array}{ll}
1 & 1 \\
0 & 1
\end{array}\right), \quad u=\left(\begin{array}{rr}
-1 & 0 \\
0 & 1
\end{array}\right) .
$$

\footnotetext{
1 It is also possible to check directly that the Bloch function associated with this function vanishes at points $k=0$ and $\pi$
} 
That $G$ is a crystallographic group in one dimension can be seen from the action of these matrices on the real line. If $x$ is a real number, we have

$$
t\left(\begin{array}{l}
x \\
1
\end{array}\right)=\left(\begin{array}{c}
x+1 \\
1
\end{array}\right), \quad u\left(\begin{array}{l}
x \\
1
\end{array}\right)=\left(\begin{array}{r}
-x \\
1
\end{array}\right),
$$

which shows that $t$ generates translations and $u$ is an inversion.

An alternative but more abstract definition is furnished by the following presentation:

$$
G=\left\langle t, u \mid u^{2}=1,(t u)^{2}=1\right\rangle .
$$

It means that elements of the group are classes of words of the type $t^{a} u^{b} t^{c} u^{d}, \ldots$ where the exponents $a, b, c, d, \ldots$ are integers of arbitrary signs. The use of the two relations $u^{2}=1$ and $(t u)^{2}=1$ permits to transform a word into a word of the same class. One sees that the relation $u^{2}=1$ permits to write any group element in the form $t^{a} u t^{c} u \ldots$. The property $t^{a} u=u t^{-a}$ can be used to show that the elements can be put ${ }^{2}$ in one of the two forms $t^{a}$ or $u t^{a}$. It is clear that the two definitions of the group are equivalent. Indeed, the elements $t^{a}$ and $u t^{a}$ are represented by the matrices $t^{a}=\left(\begin{array}{ll}1 & a \\ 0 & 1\end{array}\right), u t^{a}=\left(\begin{array}{rr}-1 & -a \\ 0 & 1\end{array}\right)$, respectively.

2. A Property Common to All Unitary Representations of the Group. Consider an arbitrary unitary representation where $t$ is represented by $T$ and $u$ by $U$. We have, obviously,

$$
T^{*}=T^{-1}, \quad U^{*}=U^{-1}, \quad U^{2}=I, \quad(T U)^{2}=I .
$$

Define

$$
P=\frac{I+U}{2} \text { and } Q=\frac{I+T U}{2} .
$$

Proposition. $P$ and $Q$ are Hermitian projections. Conversely, given two Hermitian projections $P$ and $Q$ on a Hilbert space (finite of infinite dimensional), the operators

$$
T=(2 Q-I)(2 P-I) \text { and } U=2 P-I
$$

generate a unitary representation of the group $G$.

Proof. That $P$ and $Q$ are Hermitian projections follow directly from their definition (3.5) and Eq.(3.4). Conversely, if $P$ and $Q$ are Hermitian projections, the operators $T$ and $U$ defined by (3.6) satisfy the properties (3.4).

3. Unitary Irreducible Representations. First Method. There is a direct method to obtain these representations. The one we are describing here is analogous to the one used in Part 5 for the group $F 222$. Let $|k\rangle$ be an eigenvector of $T$ associated with the eigenvalue $\exp (-i k)$, where $k$ is an angle,

$$
T|k\rangle=\exp (-i k)|k\rangle \text {. }
$$

From (3.4), $T U=U T^{-1}$, which gives

$$
T U|k\rangle=\exp (i k) U|k\rangle .
$$

\footnotetext{
2 The trivial one-dimensional space group has the presentation $G=\langle t\rangle$. It is a free group
} 
It follows that $U|k\rangle$ is also an eigenvector of $T$. The corresponding eigenvalue is different from the original one, except if $k$ is a multiple of $\pi$. For convenience, we will make the choice $-\pi<k \leq \pi$.

1. The general case $(k \neq 0, \pi)$ : We can always write $U|k\rangle=|-k\rangle$. The vectors $|k\rangle$ and $|-k\rangle$ are linearly independent. One obtains in this way a two-dimensional irreducible representation denoted $D^{(\kappa)}$, where $\kappa$. denotes the absolute value of $k(0<\kappa<\pi)$. In the basis $|\kappa\rangle,|-\kappa\rangle$, we get the matrices

$$
D^{(\kappa) \cdot}(t)=T=\left(\begin{array}{cc}
\exp (-i \kappa) & 0 \\
0 & \exp (i \kappa)
\end{array}\right), \quad D^{(\kappa)}(u)=U=\left(\begin{array}{ll}
0 & 1 \\
1 & 0
\end{array}\right) .
$$

2. $\kappa=0$. Since $U^{2}=I$, we arrive at the following pair of one-dimensional representations:

$$
\begin{array}{ll}
D^{(0,+)}(t)=1, & D^{(0,+)}(u)=1, \\
D^{(0,-)}(t)=1, & D^{(0,-)}(u)=-1 .
\end{array}
$$

3. $\kappa=\pi$. We arrive at the following pair of one-dimensional representations:

$$
\begin{array}{ll}
D^{(\pi,+)}(t)=-1, & D^{(\pi,+)}(u)=1, \\
D^{(\pi,-)}(t)=-1, & D^{(\pi,-)}(u)=-1 .
\end{array}
$$

In order to see what is going on about irreducible representations when $\kappa$ runs from zero to $\pi$, it is better to "unify" the results in choosing an equivalent representation for which $U$ is diagonal. One obtains, for an arbitrary value of $\kappa$,

$$
D^{(\kappa)}(t)=T=\left(\begin{array}{cc}
\cos \kappa & -i \sin \kappa \\
-i \sin \kappa & \cos \kappa
\end{array}\right), \quad D^{(\kappa)}(u)=U=\left(\begin{array}{rr}
1 & 0 \\
0 & -1
\end{array}\right) .
$$

For $\kappa$ taking one of the values 0 or $\pi$, the two-dimensional representation reduces into two inequivalent one dimensional representations.

Remark. We must underline that the variable $k$ labels the unirreps of the translation subgroup of $G$ although the variable $\kappa$ labels the unirreps of the group itself. Solid state physicists must have the feeling that this new labelling brings nothing new. However, we must remind the reader that we are interested here, not in representations in the strict sense, but in the classes of equivalent representations of the group $G$, a concept which is difficult to describe with the aid of the quasi-momentum alone.

4. Unitary Irreducible Representations. The Projection Method. For irreducible representations (unirreps), the projections $P$ and $Q$ can only be of rank zero or one and we readily see that the representations are of dimension 1 or 2 .

1. Unirreps of dimension 1 . We have the four following cases:

$$
\begin{array}{lll}
P=1 & Q=1 & \text { representation } D^{(0,+)} \\
P=0 & Q=0 & \text { representation } D^{(0,-)} \\
P=1 & Q=0 & \text { representation } D^{(\pi,+)} \\
P=0 & Q=1 & \text { representation } D^{(\pi,-)}
\end{array}
$$


2. Unirreps of dimension 2. We can always choose $P=\left(\begin{array}{ll}1 & 0 \\ 0 & 0\end{array}\right)$ and take, for $Q$, the most general independent projection of rank one. It can be written

$$
Q=\frac{1}{2}\left(\begin{array}{cc}
1+\cos \kappa & i \sin \kappa \\
-i \sin \kappa & 1-\cos \kappa
\end{array}\right) \text { with } \kappa \neq 0, \pi
$$

It is a simple exercise to show that the projections

$$
\Pi_{n}=\frac{1}{2}\left(\begin{array}{cc}
1+\cos (n \kappa) & i \sin (n \kappa) \\
-i \sin (n \kappa) & 1-\cos (n \kappa)
\end{array}\right)
$$

are such that

$$
\begin{aligned}
\Pi_{2 n} & =T^{n} P T^{n}, \\
\Pi_{2 n+1} & =T^{n} Q T^{n} .
\end{aligned}
$$

This furnishes an interpretation of the projections.

5. Dual of the Group G. Equations (13) contain all information needed to construct the dual of the group. For $\kappa$ lying in the open interval $] 0, \pi[$, the representation is twodimensional. When it takes the value 0 or $\pi$, we get two representations of dimension one. It follows that the dual can be represented symbolically by the diagram of Fig. 1: an open part and its closure represented symbolically by two forks of zero length.

At this stage, it is worthwhile to underline the method we have adopted for the construction of the dual. We constructed a two-dimensional matrix for the generator $t$ of the group. This matrix depends on one parameter $\kappa$ labelling all the unirreps. For $\kappa$ taking one of the values 0 or $\pi$, the representation splits into two onedimensional non-equivalent representations. We will see later how such a construction can be generalized for all crystallographic groups. Actually, that we deal with a single parameter $\kappa$ is due to the fact that we are studying a one-dimensional space group.

We already mentioned the advantage of introducing the notion of the dual of a crystallographic group. For 3-dimensional crystallographic groups, the Brillouin torus is three-dimensional and cannot be drawn easily, except if we accept to get in mind the identification of opposite faces. We will see that, in contradistinction to the Brillouin torus, the dual of the group $F 222$ can be easily drawn in a three dimensional space without the use of point identification. Generally, in order to go from the Brillouin torus to the dual of a group, two kinds of operations must be performed. (1) One must identify points of the Brillouin torus which belong to the same irreducible representation. In the present case, one identifies $k$ with $-k$ whenever $k$ is different from 0 or $\pi$. (2) One must show clearly which parts of the dual cannot be separated. In the present example, it is the case, in the one hand for the representations $D^{(0,+)}$ and $D^{(0,-)}$ and in the second hand for the representations $D^{(\pi,+)}$ and $D^{(\pi,-)}$. That they

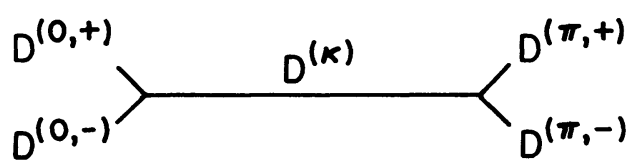

Fig. 1. The dual of $G$. $\kappa$ varies from 0 (left) to $\pi$ (right) 
cannot be separated means that, in the dual, every neighborhood of $D^{(0,+)}$ intersects any neighborhood of $D^{(0,-)}$.

In our example, we see that the dual is composed of a manifold (the open interval) with a non-standard boundary. This is the general case ${ }^{3}$. The dual of any $n$-dimensional crystallographic group always contains a manifold of dimension $n$; its boundary is complex and, more surprising, is not necessarily of dimension $n-1$.

As a last remark, we note that the representation of dimension two defined by Eqs.(3.13) can be said to be irreducible almost everywhere on the dual.

6. A Property of all Representations of $G$. Any representation (finite dimensional or infinite dimensional) of a crystallographic group is unitary and can be decomposed in a unique way into irreducible representations. This means that the unit operator can be considered as a direct integral plus a direct sum of projections in such a way that the restriction of the representation to any subspace associated with a projection is irreducible. In our simple example, this means that we have a formula of the type

$$
I=\int_{\kappa_{1}}^{\kappa_{2}} P^{(\kappa)} d \kappa+\int_{\kappa_{3}}^{\kappa_{4}} P^{(\kappa)} d \kappa+\cdots+P^{(m)}+P^{(n)}+\cdots,
$$

where $0 \leq \kappa, m, n \leq \pi$.

Obviously, two representations for which these decomposition are different are inequivalent. The converse is not necessarily true, as we will see in the next part.

The remark made about Eq.(2.3) is also valid for Eq.(3.14). The representations associated with the values $\kappa=0$ and $\pi$ can only appear in the direct sum. This subtle difference between the two cases will be examinated later on.

7. Representations for which the Hilbert Space is made of Functions on the Ordinary One-Dimensional Space. We have in mind the dual action of the one defined by Eq.(3.2), that is:

$$
\begin{aligned}
& (T f)(x)=f(x-1), \\
& (U f)(x)=f(-x) .
\end{aligned}
$$

In that case, the operators $P$ and $Q$ have a simple interpretation:

$P$ projects on the space of even functions with respect to $x=0$,

$I-P$ projects on the space of $o d d$ functions with respect to $x=0$,

$Q$ projects on the space of even functions with respect to $x=\frac{1}{2}$,

$I-Q$ projects on the space of $o d d$ functions with respect to $x=\frac{1}{2}$.

More generally,

$\Pi_{2 n}=T^{n} P T^{n}$ projects on the space of even functions with respect to $x=n$,

$\Pi_{2 n+1}=T^{n} Q T^{n}$ projects on the space of even functions with respect to $x=n+\frac{1}{2}$

8. Unirreps of the Associated Born-von Karman Groups. They are the groups $G_{N}$ $(N>2)$ defined by the same generators $t$ and $u$ of Eq.(3.1), with the restriction

\footnotetext{
3 The only exceptions concern the trivial crystallographic groups, that is the pure translation groups.
} For them, the dual has no boundary (Brillouin torus) 
that the matrix entries are defined modulo $N$. It follows that the group $G_{N}$ has $2 N$ elements. These elements are $t^{n}$ and $u t^{n}$, where $n$ takes the values $0,1,2, \ldots, N-1$.

An equivalent way of defining the group is to give the following presentation:

$$
G_{N}=\left\langle t, u \mid u^{2}=1,(t u)^{2}=1, t^{N}=1\right\rangle .
$$

We do not give here the construction of the unirreps of this group. We only want to give the following results.

a) For $N$ even but different from zero, the dual of $G_{N}$ contains the four onedimensional unirreps $D^{(0,+)}, D^{(0,-)}, D^{(\pi,+)}, D^{(\pi,-)}$ and $\frac{N}{2}-1$ two-dimensional unirreps.

b) For $N$ odd, the dual of $G_{N}$ contains the two one-dimensional unirreps $D^{(0,+)}$, $D^{(0,-)}$ and $\frac{N-1}{2}$ two-dimensional unirreps . $D^{(0,-)}$.

c) The unirreps of the point group $(N=0)$ are described by the points $D^{(0,+)}$ and

9. The Wigner-Seitz Torus and the Wyckoff Positions. From Eq.(3.2), we derive the following action of $G$ on the real line:

$$
t^{n} x=x+n, \quad u t^{n} x=-x-n,
$$

It follows that we have three kinds of points:

1) the generic points (any point $x$ such that $2 x$ is not integer) for which the little group is the trivial group $\{I\}$,

2) integral points. The little group of point $x=n$ is $\left\{I, u t^{-2 n}\right\}$, conjugate to the little group $\{I, u\}$.

3) half-integral points. The little group is $\left\{I, u t^{-n-1}\right\}$, conjugate to $\left\{I, u t^{-1}\right\}$. Indeed, $u t^{-2 n-1}=t^{n}\left(u t^{-1}\right) t^{-n}$.

We classify in this way the points of the real line in three categories, each category being called a Wyckoff position. We note that, in contradistinction with what is done in crystallography literature, our definition is pure group theoretical in that we are not making use of the notion of Euclidean distance. The word position can be better understood in introducing the Wigner-Seitz torus. This torus is obtained in identifying all points of the real line which have the same fractional part. The Wigner-Seitz torus is, in our case, a circle. This circle has three Wyckoff positions: the generic one, the integral one (denoted 0 ), and the half-integral one (denoted $\frac{1}{2}$ ). With each Wyckoff position corresponds a class of conjugate subgroups. There is an open Wyckoff position represented by the generic point and two closed positions, namely the ones represented by 0 and $\frac{1}{2}$. This property is discussed in $[7,15]$.

10. Band Representations. Instead of looking at the action of $G$ on the real line, one can make $G$ acting on a localized ${ }^{4}$ function $f(x)$ on the real line in the way described by Eqs.(3.15) and (3.16). They are the quasi-band representations. The action of the group introduces the following functions:

$$
\begin{aligned}
& \left(T^{n}\right) f(x)=f\left(t^{-n} x\right)=f(x-n), \\
& \left(U T^{n}\right) f(x)=f\left(t^{-n} u x\right)=f(-x-n) .
\end{aligned}
$$

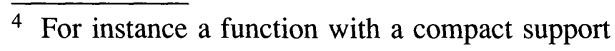


Suppose, for simplicity, that the function we start with has a compact support of length less than one. In that case we are certain that we deal with a band representation (i.e. an induced representation) and we have the three following cases.

1) Suppose that the function $f$ we start with has no symmetry (that is the group of symmetry of $f$ is trivial, the little group of $f$ being the one of the first Wyckoff position). Suppose, moreover that its Fourier transform has no zero. Then, each element of the group applied to $f$ provides us with a distinct function. The functions obtained in this way span a space on which the group $G$ acts. This representation is the band representation induced from the trivial representation of the trivial group 1, namely the little group associated with the generic Wyckoff position. It is equivalent to the regular representation. Since we are interested in equivalence classes of representations, we will denote it by $\Gamma^{(\mathrm{reg})}$. The regular representation is described simply by the action of the generators on the basis functions $f_{n}^{+}(x)=f(x-n)$ and $f_{n}^{-}(x)=f(-x-n)$,

$$
\begin{array}{ll}
T f_{n}^{+}=f_{n+1}^{+}, & T f_{n}^{-}=f_{n-1}^{-}, \\
U f_{n}^{+}=f_{n}^{-}, & U f_{n}^{-}=f_{n}^{+} .
\end{array}
$$

When the function $f$ has a given symmetry, we get band representations of particular types. To classify these types, we have to say how the function $f$ behaves under one of the little groups of the non-trivial Wyckoff positions.

2) Wyckoff position 0: two cases occur. The function $f$ either is invariant or change sign under one of the little groups of this Wyckoff positions. This means that we have, for instance,

$$
f(-x)= \pm f(x) .
$$

The plus sign corresponds to the representation $\gamma^{(0,+)}$ of the little group, for which

$I$ is represented by multiplication by 1 ,

$u$ is also represented by multiplication by 1 .

The minus sign corresponds to the representation $\gamma^{(0,-)}$ of the little group, for which

$$
\begin{aligned}
& I \text { is represented by multiplication by } 1, \\
& u \text { is represented by multiplication by }-1 \text {. }
\end{aligned}
$$

These two representations are of dimension one. They induce band representations $\Gamma^{(0,+)}$ and $\Gamma^{(0,-)}$, respectively. ${ }^{5}$

3) Wyckoff position $\frac{1}{2}$ : The discussion is analogous to the one of case 2 . We note that the class of subgroups is now different but isomorphic to the groups of case 2 . The function $f$ can be even or odd with respect to one of these groups. For instance, $f(1-x)= \pm f(x)$. We arrive at band representations $\Gamma^{(1 / 2,+)}$ and $\Gamma^{(1 / 2,-)}$, induced from the unirreps $\gamma^{(1 / 2,+)}$ and $\gamma^{(1 / 2,-)}$, respectively.

In cases 2 and 3 , the band representations are said to be elementary or (improperly) irreducible because if we start with an arbitrary function $f$, we can always write it as a linear combination of an even part and an odd part relatively to an arbitrary symmetry point [12]. We note that elementary band representations are associated with closed Wyckoff positions. These elementary representations are subrepresentations of the regular representation.

5 It is perhaps necessary to insist: the word representation here means class of equivalent representations 
We will examine later on the case of quasi-band representations which are not induced representations since the group action on the function $f$ gives rise to linearly dependent functions.

11. Decomposition of the Band Representations. Here we will show that the five band representations of the group $G$ which we have denoted $\Gamma^{(\mathrm{reg})}, \Gamma^{(0,+)} \Gamma^{(0,-)}$, $\Gamma^{(1 / 2,+)}$, and $\Gamma^{(1 / 2,-)}$ have different contents and, consequently, are not equivalent. The contents are described by the diagrams of Fig. 2 .

a) We start with the regular representation $\Gamma^{(\mathrm{reg})}$. The representation space is spanned by the functions $f_{n}^{ \pm}$. We look for the linear combinations

$$
\psi_{k}(x)=\sum_{-\infty}^{+\infty} \alpha_{n}^{(k)} f(x-n)+\sum_{-\infty}^{+\infty} \beta_{n}^{(k)} f(-x-n)
$$

which are eigenfunctions of the operator $T$ with eigenvalue $e^{-i k}$. We get, with the aid of a change of the summation index,

$$
T \psi_{k}(x)=\sum_{-\infty}^{+\infty} \alpha_{n-1}^{(k)} f(x-n)+\sum_{-\infty}^{+\infty} \beta_{n+1}^{(k)} f(-x-n)
$$

which provides us with the conditions

$$
\alpha_{n-1}^{(k)}=e^{-i k} \alpha_{n}^{(k)}, \quad \beta_{n+1}^{(k)}=e^{-i k} \beta_{n}^{(k)} .
$$

This shows that $\psi_{k}(x)$ is a linear combination of the two Bloch functions

$$
\sum_{-\infty}^{+\infty} e^{\imath n k} f(x-n) \text { and } \sum_{-\infty}^{+\infty} e^{-i n k} f(-x-n) .
$$

Define the following combination:

$$
\psi_{\kappa}(x)=\alpha \sum_{-\infty}^{+\infty} e^{i n \kappa} f(x-n)+\beta \sum_{-\infty}^{+\infty} e^{-i n \kappa} f(-x-n)
$$

with $0 \leq \kappa \leq \pi$. We have $T \psi_{\kappa}(x)=e^{-i \kappa} \psi_{\kappa}(x)$. In order to build the representation (3.8), we have to introduce the other function

$$
U \psi_{\kappa}(x)=\alpha \sum_{-\infty}^{+\infty} e^{i n \kappa} f(-x-n)+\beta \sum_{-\infty}^{+\infty} e^{-i n \kappa} f(x-n)
$$

We go to the representation (3.13) in taking the two basis functions

$$
\psi_{\kappa}^{ \pm}(x)=\psi_{\kappa}(x) \pm U \psi_{\kappa}(x)
$$

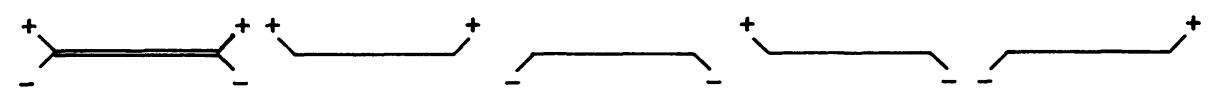

Fig. 2. The general contents of band representations. $\kappa$ varies from 0 (left) to $\pi$ (right) 
The action of the generators $T$ and $U$ is given by the following equations:

$$
\begin{aligned}
& T \psi_{\kappa}^{(+)}(x)=\cos \kappa \psi_{\kappa}^{(+)}(x)-i \sin \kappa \psi_{\kappa}^{(-)}(x) \\
& T \psi_{\kappa}^{(-)}(x)=-i \sin \kappa \psi_{\kappa}^{(+)}(x)+\cos \kappa \psi_{\kappa}^{(-)}(x) \\
& U \psi_{\kappa}^{(+)}(x)=\psi_{\kappa}^{(+)}(x) \\
& U \psi_{\kappa}^{(-)}(x)=-\psi_{\kappa}^{(-)}(x)
\end{aligned}
$$

Since the coefficients $\alpha$ and $\beta$ are arbitrary, each representation $D^{(\kappa)}$ is obtained twice. When we go to the limit cases $\kappa=0$ or $\pi$, we get

$$
\begin{aligned}
\psi_{0}^{( \pm)}(x) & =\psi_{0}(x) \pm U \psi_{0}(x) \\
& =\alpha \sum_{-\infty}^{+\infty} f(x-n)+\beta \sum_{-\infty}^{+\infty} f(-x-n) \pm \alpha \sum_{-\infty}^{+\infty} f(-x-n) \pm \beta \sum_{-\infty}^{+\infty} f(x-n) \\
& =(a \pm \beta) \sum_{-\infty}^{+\infty}[f(x-n) \pm f(-x-n)]
\end{aligned}
$$

and, similarly,

$$
\psi_{\pi}^{( \pm)}(x)=(a \pm \beta) \sum_{-\infty}^{+\infty}(-)^{n}[f(x-n) \pm f(-x-n)] .
$$

This shows that we get each representation $D^{(0,+)}, D^{(0,-)}, D^{(\pi,+)}$ and $D^{(\pi,-)}$ only once. This result is shown on the first diagram of Fig. 2.

Let us now examine the representations $\Gamma^{(0, \varepsilon)}$ for which the function $f$ is even $(\varepsilon=1)$ or odd $(\varepsilon=-1)$ with respect to an arbitrary integral point (the origin, for convenience). We set

$$
f(x)=\varepsilon f(-x)
$$

Equation (3.18) simplifies

$$
\psi_{\kappa}(x)=(\alpha+\varepsilon \beta) \sum_{\infty}^{+\infty} e^{i n \kappa} f(x-n) .
$$

It proves that each representation $D^{(\kappa)}$ appears only once. Equations (3.20) and (3.21) now read, up to a factor

$$
\begin{aligned}
& \psi_{0}^{( \pm)}(x)=(1 \pm \varepsilon) \sum_{-\infty}^{+\infty} f(x-n), \\
& \psi_{\pi}^{( \pm)}(x)=(1 \mp \varepsilon) \sum_{-\infty}^{+\infty}(-)^{n} f(x-n) .
\end{aligned}
$$

A simple discussion permits to obtain the other diagrams of Fig. 2.

Since the five representations we arrived at have different unirreps contents, we are sure that they cannot be equivalent. For the moment, we could check directly 
that the representations built on an even function $f(x)$ and the translated function $g(x)=f(x-1 / 2)$ are inequivalent, that is they cannot be intertwined. ${ }^{6}$

We know already how the generators $T$ and $U$ act in the case of an even function $f$. It is easy to see that the action of $T$ is the same on the set of functions $\psi_{\kappa}^{(+)}(x)$ and $\psi_{\kappa}^{(-)}(x)$ built on $f$ and on the corresponding set $\phi_{\kappa}^{(+)}(x)$ and $\phi_{\kappa}^{(-)}(x)$ which is built on $g$. Let us examine the action of $U$ on $\phi_{\kappa}^{(+)}(x)$ and $\phi_{\kappa}^{(-)}(x)$,

$$
\begin{aligned}
U \phi_{\kappa}^{(+)}(x) & =U \sum e^{i n \kappa}\left[f\left(x-n-\frac{1}{2}\right)+f\left(-x-n+\frac{1}{2}\right)\right] \\
& =\sum e^{i n \kappa}\left[f\left(-x-n-\frac{1}{2}\right)+f\left(x-n+\frac{1}{2}\right)\right] \\
& =\sum e^{i(n-1) \kappa} f\left(-x-n+\frac{1}{2}\right)+\sum e^{i(n+1) \kappa} f\left(x-n-\frac{1}{2}\right) \\
& =\cos \kappa \phi_{\kappa}^{(+)}(x)+i \sin \kappa \phi_{\kappa}^{(-)}(x) .
\end{aligned}
$$

A similar calculation can be done for the action of $U$ on $\phi_{\kappa}^{(-)}(x)$. We get finally, for the matrix $U$

$$
U=\left(\begin{array}{cc}
\cos \kappa & i \sin \kappa \\
-i \sin \kappa & -\cos \kappa
\end{array}\right)
$$

and it is easy to verify that none matrix commuting with $T$ can intertwine this matrix with the diagonal matrix $\left(\begin{array}{rr}1 & 0 \\ 0 & -1\end{array}\right)$.

Remark. The fact that, in Fig. 2, the diagram of the regular representation can be seen as the union of the diagrams of $\Gamma^{(0,+)}$ and $\Gamma^{(0,-)}$ or those of $\Gamma^{(1 / 2,+)}$ and $\Gamma^{(1 / 2,-)}$ is due to the fact that any function can be regarded as the sum of its even and odd parts.

12. Quasi-Band Representations. It is interesting to see what the four elementary band representations become when we permit the functions to be linearly dependent, that is when we turn towards quasi-band representations.

Let us examine, for instance, the case where an even function $f$ is chosen in such a way that $\psi_{\kappa}^{(+)}(x)$ vanishes at one of the limit points of the dual (and nowhere else). As an example, we take the function

$$
f(x)=-e^{-(x-1)^{2} / 2}+2 e^{-x^{2} / 2}-e^{-(x+1)^{2} / 2} .
$$

It is a simple matter to check directly ${ }^{7}$ that the function $\psi_{\kappa}^{(+)}$vanishes at point $\kappa=0$. In fact,

$$
\begin{aligned}
\psi_{\kappa}^{(+)}(x) & =\sum_{-\infty}^{+\infty} e^{-i n \kappa}\left[-e^{-(x-n-1)^{2} / 2}+2 e^{-(x-n)^{2} / 2}-e^{-(x-n+1)^{2} / 2}\right] \\
& =2(1-\cos \kappa) \sum_{-\infty}^{+\infty} e^{-i n \kappa} e^{-(x-n)^{2} / 2}
\end{aligned}
$$

\footnotetext{
$\overline{6}$ It is clear that the functions $f(x \pm 1), f(x \pm 2)$, etc. would lead to the same representation as the one built on $f(x)$. This is due to the role of the Wyckoff positions

7 We could also use the Fourier transform to prove that property
} 


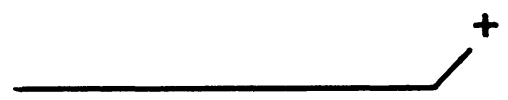

Fig. 3. The quasi-band representation associated with the function (3.22)

We already know that $\psi_{\kappa}^{(-)}(x)$ also vanishes at point $\kappa=0$. That is why we arrived in this way at a quasi-band representation which could be represented by the diagram of Fig. 3.

It is obvious that if we consider, instead of $f(x)$, the translated function $g(x)=$ $f(x-1 / 2)$, it will provide us with a quasi-band representation with the same unirreps decomposition. It is natural to examine if these two representation are equivalent. The answer is yes. The proof is very easy. We only have to perform a similar calculation with the translated function $g(x)=f(x-1 / 2)$. We get

$$
\phi_{\kappa}^{(+)}(x)=\sum_{-\infty}^{+\infty} e^{-i n \kappa}\left[-e^{-(x-n-3 / 2)^{2} / 2}+2 e^{-(x-n-1 / 2)^{2} / 2}-e^{-(x-n+1 / 2)^{2} / 2}\right],
$$

that is

$$
\phi_{\kappa}^{(+)}(x)=2 e^{-i \kappa / 2}(1-\cos \kappa) \sum_{-\infty}^{+\infty} e^{i n \kappa} e^{-(x-n)^{2} / 2}
$$

which shows directly that the functions $\psi_{\kappa}^{(+)}(x)$ and $\phi_{\kappa}^{(+)}(x)$ only differ from a phase factor.

The above calculation proves that the two representations $\Gamma^{(0,+)}$ and $\Gamma^{(1 / 2,+)}$ can be transformed into equivalent representations provided the localized function we start with is suitably chosen. The calculation we have presented for their explicit inequivalence was valid for almost all functions $f$.

We can find other types of quasi-band representations. For instance, the function which has already been considered

$$
f(x)=e^{-(x-1)^{2} / 2}-e^{-(x+1)^{2} / 2}
$$

provides us with a quasi-band representation which contains none of the limit unirreps.

We will conclude this part by an important remark. As we already saw, the unirreps decomposition of a representation is described by direct integrals and direct sums. The existence of a zero of a Bloch function on the Brillouin torus does not have the same meaning when the zero lies on a symmetry point and when it lies elsewhere. In the first case, a subunirrep is missing, in the second one, it involves the direct integral and this direct integral does not change. In order to illustrate the fact, one can consider the following family of functions parametrized by $a$ by $b$ :

$$
f(x)=2 a \exp \left(-\frac{x^{2}}{2}\right)-b\left[\exp \left(-\frac{(x+1)^{2}}{2}\right)+\exp \left(-\frac{(x-1)^{2}}{2}\right)\right] .
$$

We note that the function (3.22) belongs to this family.

The Fourier transform of (3.23) has, as a factor, the expression $a-b \cos p$. According to the values of the parameters we have the following categories of quasiband representations: 
1) $|a|>|b|$

$$
I=\int_{0}^{\pi} P^{(\kappa)} d \kappa+P^{(0,+)}+P^{(\pi,+)}
$$

it is a band representation

2) $|a|<|b|$

$$
I=\int_{0}^{\pi} P^{(\kappa)} d \kappa+P^{(0,+)}+P^{(\pi,+)}
$$

it has the same decomposition but it is not a band representation.

3) $a=b$

$$
I=\int_{0}^{\pi} P^{(\kappa)} d \kappa+P^{(\pi,+)} .
$$

4) $a=-b$

$$
I=\int_{0}^{\pi} P^{(\kappa)} d \kappa+P^{(0,+)} .
$$

Our example underlines the difficulties appearing when we examine the set of all quasi-band representations.

13. Concluding remark. Two Conjectures. The dual $G^{*}$ of the non-trivial onedimensional space group has four maximal compact subduals. Each of them is associated with a unique elementary band. We are tempted to state the following conjectures.

Conjecture 1. If $G$ is a symmorphic group, each elementary band representation which has a single branch decomposes with multiplicity one on a maximal compact subdual. Conjecture 2. Given a maximal compact subdual, there exists at least one elementary band representation associated with it.

\section{The F222 Group}

1. Definition of the group. Let us start by the recall of some general facts. A threedimensional crystallographic group $G$ can always be defined with the aid of matrices of the form

$$
g=\left(\begin{array}{cccc}
g_{11} & g_{12} & g_{13} & g_{14} \\
g_{21} & g_{22} & g_{23} & g_{24} \\
g_{31} & g_{32} & g_{33} & g_{34} \\
0 & 0 & 0 & 1
\end{array}\right)
$$

where the entries are rational numbers. We make the group acting on $X=\left(\begin{array}{c}x \\ y \\ z \\ 1\end{array}\right)$. The little group $G_{X}$ of $X$ is made of those elements $g$ which satisfy $g X=X$. The 
class $[X]$ of points $X$ which have conjugate little groups form a Wyckoff position. If we denote a class of conjugate little groups by $\left[G_{X}\right]$, we can define an order relation between these classes as follows: $\left[G_{X}\right]<\left[G_{X^{\prime}}\right]$ if there are points $X$ and $X^{\prime}$ such that $G_{X}$ is a subgroup of $G_{X^{\prime}}$. Then $\left[X^{\prime}\right]$ is contained in the closure of $[X]$.

Given a localized function $f(X)$, we can make the group acting on it as follows:

$$
(g \cdot f)(X)=f\left(g^{-1} X\right) .
$$

We obtain in this way a set of functions which provide us with a quasi-band representation or a band representation depending on the fact that those functions are linearly dependent or not.

A band representation is said to be elementary (or, but improperly, irreducible) if it cannot be reduced into other band representation. Equivalently, it is a representation which is induced from a one-dimensional unirrep of a group of the class $\left[G_{X}\right]$, where $X$ lies in a closed Wyckoff position (i.e. $G_{X}$ is a finite maximal subgroup [8]).

The group $F 222$ if generated by the six following matrices:

$$
\begin{aligned}
& t_{1}=\left(\begin{array}{cccc}
1 & 0 & 0 & 0 \\
0 & 1 & 0 & \frac{1}{2} \\
0 & 0 & 1 & \frac{1}{2} \\
0 & 0 & 0 & 1
\end{array}\right) \quad t_{2}=\left(\begin{array}{cccc}
1 & 0 & 0 & \frac{1}{2} \\
0 & 1 & 0 & 0 \\
0 & 0 & 1 & \frac{1}{2} \\
0 & 0 & 0 & 1
\end{array}\right) \quad t_{3}=\left(\begin{array}{cccc}
1 & 0 & 0 & \frac{1}{2} \\
0 & 1 & 0 & \frac{1}{2} \\
0 & 0 & 1 & 0 \\
0 & 0 & 0 & 1
\end{array}\right) \\
& u_{1}=\left(\begin{array}{rrrr}
1 & 0 & 0 & 0 \\
0 & -1 & 0 & 0 \\
0 & 0 & -1 & 0 \\
0 & 0 & 0 & 1
\end{array}\right) \quad u_{2}=\left(\begin{array}{rrrr}
-1 & 0 & 0 & 0 \\
0 & 1 & 0 & 0 \\
0 & 0 & -1 & 0 \\
0 & 0 & 0 & 1
\end{array}\right) u_{3}=\left(\begin{array}{rrrr}
-1 & 0 & 0 & 0 \\
0 & -1 & 0 & 0 \\
0 & 0 & 1 & 0 \\
0 & 0 & 0 & 1
\end{array}\right) \text {. }
\end{aligned}
$$

We have the following relations, where $a, b, c$ are integers:

$$
\begin{array}{lll}
u_{1} u_{2}=u_{2} u_{1}=u_{3}, & u_{2} u_{3}=u_{3} u_{2}=u_{1}, & u_{3} u_{1}=u_{1} u_{3}=u_{2}, \\
t_{1} t_{2}=t_{2} t_{1}, & t_{2} t_{3}=t_{3} t_{2}, & t_{3} t_{1}=t_{1} t_{3}, \\
t_{1} u_{1}=u_{1} t_{1}^{-1}, & t_{1} u_{2}=u_{2} t_{2}^{-1} t_{3}, & t_{1} u_{3}=u_{3} t_{3}^{-1} t_{2}, \\
t_{2} u_{1}=u_{1} t_{1}^{-1} t_{3}, & t_{2} u_{2}=u_{2} t_{2}^{-1}, & t_{2} u_{3}=u_{3} t_{3}^{-1} t_{1}, \\
t_{3} u_{1}=u_{1} t_{1}^{-1} t_{2}, & t_{3} u_{2}=u_{2} t_{2}^{-1} t_{1}, & t_{3} u_{3}=u_{3} t_{3}^{-1} .
\end{array}
$$

2. Closed Wyckoff Positions [1,8]. They correspond to four equivalence classes of subgroups, all isomorphic to the dihedral group $D_{2}$.

Class (a) subgroups conjugate to $\left\{1, u_{1}, u_{2}, u_{3}\right\}$,

Class $(b)$ subgroups conjugate to $\left\{1, u_{1} t_{1}^{-1} t_{2}^{-1} t_{3}, u_{2} t_{1}^{-1} t_{2}^{-1} t_{3}, u_{3}\right\}$,

Class (c) subgroups conjugate to $\left\{1, u_{1} t_{1}^{-1}, u_{2} t_{2}^{-1}, u_{3} t_{3}^{-1}\right\}$,

Class $(d)$ subgroups conjugate to $\left\{1, u_{1} t_{1}^{-2} t_{2}^{-1} t_{3}, u_{2} t_{1}^{-1} t_{2}^{-1} t_{3}, u_{3} t_{3}^{-1}\right\}$.

3. Unitary Irreducible Representations. Denote by $T_{i}$ and $U_{\imath}(i=1,2,3)$ the representatives of the elements $t_{i}$ and $u_{i}$ of the group in the most general unirrep. 
Let $\left|k_{1}, k_{2}, k_{3}\right\rangle$ one of the eigenvectors of the (commuting) operators $T_{i}$,

$$
\begin{aligned}
& T_{1}\left|k_{1}, k_{2}, k_{3}\right\rangle=\exp \left(-i k_{1}\right)\left|k_{1}, k_{2}, k_{3}\right\rangle, \\
& T_{2}\left|k_{1}, k_{2}, k_{3}\right\rangle=\exp \left(-i k_{2}\right)\left|k_{1}, k_{2}, k_{3}\right\rangle, \\
& T_{3}\left|k_{1}, k_{2}, k_{3}\right\rangle=\exp \left(-i k_{3}\right)\left|k_{1}, k_{2}, k_{3}\right\rangle,
\end{aligned}
$$

$\left(k_{1}, k_{2}, k_{3}\right.$ are supposed to run from 0 to $\left.2 \pi\right)$.

The relation $t_{1} u_{1}=u_{1} t_{1}^{-1}$ shows us that the vector $U_{1}\left|k_{1}, k_{2}, k_{3}\right\rangle$ is an eigenvector of $t_{1}$ with eigenvalue $\exp \left(i k_{1}\right)$. Similarly, the relations $t_{2} u_{1}=u_{1} t_{1}^{-1} t_{3}$ and $t_{3} u_{1}=u_{1} t_{1}^{-1}$ show that $U_{1}\left|k_{1}, k_{2}, k_{3}\right\rangle$ is an eigenvector of $T_{2}$ (eigenvalue $\exp \left(i\left(k_{1}-k_{3}\right)\right)$ ) and of $T_{3}$ (eigenvalue $\exp \left(i\left(k_{1}-k_{2}\right)\right)$ ). It follows that for almost every value of the triple $\left(k_{1}, k_{2}, k_{3}\right)$ we must introduce a new vector

$$
U_{1}\left|k_{1}, k_{2}, k_{3}\right\rangle=\left|-k_{1}, k_{3}-k_{1}, k_{2}-k_{1}\right\rangle \text {. }
$$

The other relations (4.3) provide us with similar relations, namely

$$
\begin{array}{ll}
U_{2} \mid k_{1}, & \left.k_{2}, k_{3}\right\rangle=\left|k_{3}-k_{2},-k_{2}, k_{1}-k_{2}\right\rangle, \\
U_{3} \mid k_{1}, & \left.k_{2}, k_{3}\right\rangle=\left|k_{2}-k_{3}, k_{1}-k_{3},-k_{3}\right\rangle .
\end{array}
$$

We are led to examine the set of quadruples of triples

$$
\left\{\left(k_{1}, k_{2}, k_{3}\right),\left(-k_{1}, k_{3}-k_{1}, k_{2}-k_{1}\right),\left(k_{3}-k_{2},-k_{2}, k_{1}-k_{2}\right),\left(k_{2}-k_{3}, k_{1}-k_{3},-k_{3}\right)\right\} \text {. }
$$

Such a quadruple is known as a star in the Brillouin torus.

Among stars, there are "ordinary" stars made of distinct triples and "symmetric"stars made of two distinct triples or for which all triples are the same. We are interested first in ordinary stars. For that, we divide the Brillouin torus (represents on Fig. 4) in six parts $B_{i j k}$ defined as follows:

$$
B_{i j k} \text { contains all triples for which } 2 \pi>k_{i}>k_{j}>k_{k}>0 \text {. }
$$

Each of $B_{i j k}$ can be divided in

$$
\begin{aligned}
& B_{i j k}^{+} \text {for which } k_{j}>2 \pi, \\
& B_{i j k}^{-} \text {for which } k_{j}<2 \pi .
\end{aligned}
$$

The union $V$ of the three parts $B_{123}^{-}, B_{231}^{-}, B_{312}^{-}$has the property that each ordinary star has one triple in $V$ and, conversely, each triple of $V$ belongs to an ordinary star. In fact, if $\left(k_{1}, k_{2}, k_{3}\right)$ belongs to $B_{123}^{-}$, the triples $\left(2 \pi-k_{1}, 2 \pi+k_{3}-k_{1}, 2 \pi+k_{2}-k_{1}\right)$, $\left(2 \pi+k_{3}-k_{2}, 2 \pi-k_{2}, k_{1}-k_{2}\right),\left(k_{2}-k_{3}, k_{1}-k_{3}, 2 \pi-k_{3}\right)$ belong respectively to $B_{321}, B_{123}^{+}, B_{321}$ that is, lie outside $V$.

It follows that the open set $V$ describe the open part of the dual of the group. It is a dense subset of the set of all unirreps of dimension four. This open set is represented on Fig. 5.

Before analyzing the symmetric stars, which correspond to the boundary of $V$, we will describe the general four-dimensional unirrep. In supposing that $\left(k_{1}, k_{2}, k_{3}\right)$ 


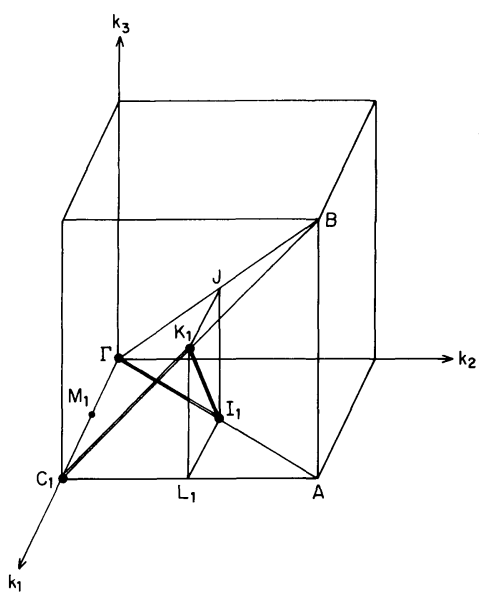

Fig. 4

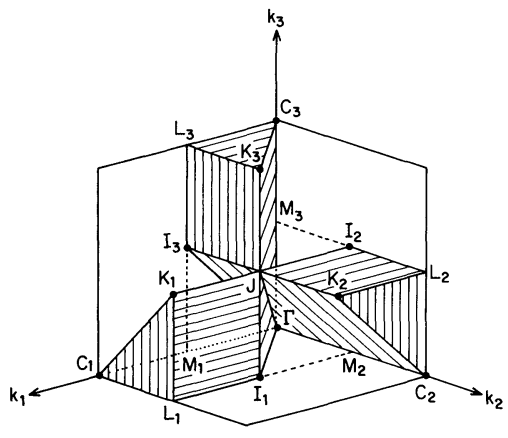

Fig. 5

Fig. 4. The Brillouin torus of the group $F$ 222. Opposite faces of the parallelepiped must be identified. The part $B_{123}^{-}$is the solid limited by the faces $J \Gamma C_{1} K_{1}, J \Gamma I_{1}, J K_{1} L_{1} I_{1}, K_{1} L_{1} C_{1}$, and $\Gamma I_{1} L_{1} C_{1}$

Fig. 5. The open set $V$ is the union of $B_{123}^{-}, B_{231}^{-}$and $B_{312}^{-}$

belongs to $B_{123}^{-}$, we can write it in the matrix form as follows:

$$
\begin{aligned}
& T_{1}=\left(\begin{array}{cccc}
\exp \left(-i k_{1}\right) & 0 & 0 & 0 \\
0 & \exp \left(i k_{1}\right) & 0 & 0 \\
0 & 0 & \exp \left(i\left(k_{2}-k_{3}\right)\right) & 0 \\
0 & 0 & 0 & \exp \left(i\left(k_{3}-k_{2}\right)\right)
\end{array}\right) \\
& T_{2}=\left(\begin{array}{cccc}
\exp \left(-i k_{2}\right) & 0 & 0 & 0 \\
0 & \exp \left(i\left(k_{1}-k_{3}\right)\right) & 0 & 0 \\
0 & 0 & \exp \left(i k_{2}\right) & 0 \\
0 & 0 & 0 & \exp \left(i\left(k_{3}-k_{1}\right)\right)
\end{array}\right) \text {, } \\
& T_{3}=\left(\begin{array}{cccc}
\exp \left(-i k_{3}\right) & 0 & 0 & 0 \\
0 & \exp \left(i\left(k_{1}-k_{2}\right)\right) & 0 & 0 \\
0 & 0 & \exp \left(i\left(k_{2}-k_{1}\right)\right) & 0 \\
0 & 0 & 0 & \left.\exp \left(i k_{3}\right)\right)
\end{array}\right) \text {, } \\
& U_{1}=\left(\begin{array}{llll}
0 & 1 & 0 & 0 \\
1 & 0 & 0 & 0 \\
0 & 0 & 0 & 1 \\
0 & 0 & 1 & 0
\end{array}\right) \quad U_{2}\left(\begin{array}{llll}
0 & 0 & 1 & 0 \\
0 & 0 & 0 & 1 \\
1 & 0 & 0 & 0 \\
0 & 1 & 0 & 0
\end{array}\right) \quad U_{3}=\left(\begin{array}{llll}
0 & 0 & 0 & 1 \\
0 & 0 & 1 & 0 \\
0 & 1 & 0 & 0 \\
1 & 0 & 0 & 0
\end{array}\right)
\end{aligned}
$$

In order to simplify the investigation of the topology of the dual, it is convenient to choose a new basis for which the $U_{i^{\prime}} s$ are diagonal. For this purpose, we perform the 
transformation defined by the following matrix:

$$
M=\frac{1}{2}\left(\begin{array}{rrrr}
1 & 1 & -1 & -1 \\
1 & -1 & 1 & -1 \\
1 & -1 & -1 & 1 \\
1 & 1 & 1 & 1
\end{array}\right)
$$

(the inverse of $M$ is its transposed). We now get

$$
\begin{gathered}
U_{1}=\left(\begin{array}{rrrr}
1 & 0 & 0 & 0 \\
0 & -1 & 0 & 0 \\
0 & 0 & -1 & 0 \\
0 & 0 & 0 & 1
\end{array}\right) \quad U_{2}=\left(\begin{array}{rrrr}
-1 & 0 & 0 & 0 \\
0 & 1 & 0 & 0 \\
0 & 0 & -1 & 0 \\
0 & 0 & 0 & 1
\end{array}\right) \\
U_{3}=\left(\begin{array}{rrrr}
-1 & 0 & 0 & 0 \\
0 & -1 & 0 & 0 \\
0 & 0 & 1 & 0 \\
0 & 0 & 0 & 1
\end{array}\right), \\
T_{1}=\frac{1}{2}\left(\begin{array}{cccc}
c_{1}+c_{23} & -i s_{1}-i s_{23} & -i s_{1}+i s_{23} & c_{1}-c_{23} \\
-i s_{1}-i s_{23} & c_{1}+c_{23} & c_{1}-c_{23} & -i s_{1}+i s_{23} \\
-i s_{1}+i s_{23} & c_{1}-c_{23} & c_{1}+c_{23} & -i s_{1}-i s_{23} \\
c_{1}-c_{23} & -i s_{1}+i s_{23} & -i s_{1}-i s_{23} & c_{1}+c_{23}
\end{array}\right) \\
T_{2}=\frac{1}{2}\left(\begin{array}{cccc}
c_{2}+c_{31} & -i s_{2}+i s_{31} & c_{2}-c_{31} & -i s_{2}-i s_{31} \\
-i s_{2}+i s_{31} & c_{2}+c_{31} & -i s_{2}-i s_{31} & c_{2}-c_{31} \\
c_{2}-c_{31} & -i s_{2}-i s_{31} & c_{2}+c_{31} & -i s_{2}+i s_{31} \\
-i s_{2}-i s_{31} & c_{2}-c_{31} & -i s_{2}+i s_{31} & c_{2}+c_{31}
\end{array}\right) \\
T_{3}=\frac{1}{2}\left(\begin{array}{cccc}
c_{3}+c_{12} & c_{3}-c_{12} & -i s_{3}-i s_{12} & -i s_{3}+i s_{12} \\
c_{3}-c_{12} & c_{3}+c_{12} & -i s_{3}+i s_{12} & -i s_{3}-i s_{12} \\
-i s_{3}-i s_{12} & -i s_{3}+i s_{12} & c_{3}+c_{12} & c_{3}-c_{12} \\
-i s_{3}+i s_{12} & -i s_{3}-i s_{12} & c_{3}-c_{12} & c_{3}+c_{12}
\end{array}\right)
\end{gathered}
$$

where $c_{i}, s_{i}, c_{i j}, s_{i j}$ are put for $\cos k_{i}, \sin k_{i}, \cos \left(k_{i}-k_{j}\right), \sin \left(k_{i}-k_{j}\right)$, respectively.

We examine now what happens to the representation when we arrive on the boundary of $B_{123}^{-}$. In general, three kinds of things may occur. Case $a$. The representation reduces into equivalent unirreps. There is no bifurcation analogous to the forks we got in Fig. 1 for the infinite dihedral group. Case $b$. The representation reduces into an irreducible one of lower dimension (some matrix entries vanishes); here too, we have no bifurcation. Case $c$. The representation reduces into inequivalent unirreps. Then a "non-separated fact" takes place, as in Fig. 1. Obviously, there is also a fourth case for which nothing happens.

It is easy to see that the only changes happen on the broken line $\Gamma I_{1} K_{1} C_{1}$. This line is closed since, because of the identification of opposite faces in the Brillouin zone, the points $\Gamma$ and $C_{1}$ are identical. The situation is as follows.

a) Point $\Gamma\left(k_{1}=k_{2}=k_{3}=0\right)$. We must emphasize that this point is the dual of the point group, that is the quotient group $G / T$, where $T$ is the whole lattice translation 
subgroup. This group is isomorphic to $D_{2}$. It is Abelian and has four elements. That is why it provides us with four one-dimensional unirreps.

The three generators $T_{i}$ are (obviously) trivially represented by 1 . We get the four following one-dimensional unirreps labelled by the corresponding eigenvalues of the $U_{i}$ 's.

$$
\begin{array}{llll}
D_{(+,-,-)}^{(\Gamma)} & U_{1}=+1 & U_{2}=-1 & U_{3}=-1 \\
D_{(-,+,-)}^{(\Gamma)} & U_{1}=-1 & U_{2}=+1 & U_{3}=-1, \\
D_{(-,-,+)}^{(\Gamma)} & U_{1}=-1 & U_{2}=-1 & U_{3}=+1 \\
D_{(+,+,+)}^{(\Gamma)} & U_{1}=+1 & U_{2}=+1 & U_{3}=+1 .
\end{array}
$$

The last representation is the trivial one.

b) Point $I_{1}\left(k_{1}=k_{2}=\pi, k_{3}=0\right)$. We have $T_{1}=T_{2}=-1, T_{3}=+1$ and we arrived at the representations

$$
\begin{array}{llll}
D_{(+,-,-)}^{\left(I_{1}\right)} & U_{1}=+1 & U_{2}=-1 & U_{3}=-1 \\
D_{(-,+,-)}^{\left(I_{1}\right)} & U_{1}=-1 & U_{2}=+1 & U_{3}=-1 \\
D_{(-,-,+)}^{\left(I_{1}\right)} & U_{1}=-1 & U_{2}=-1 & U_{3}=+1 \\
D_{(+,+,+)}^{\left(I_{1}\right)} & U_{1}=+1 & U_{2}=+1 & U_{3}=+1 .
\end{array}
$$

c) Point $K_{1}\left(k_{1}=2 \pi, k_{2}=k_{3}=\pi\right)$

$$
\begin{array}{llll}
D_{(+,-,-)}^{\left(K_{1}\right)} & U_{1}=+1 & U_{2}=-1 & U_{3}=-1, \\
D_{(-,+,-)}^{\left(K_{1}\right)} & U_{1}=-1 & U_{2}=+1 & U_{3}=-1, \\
D_{(-,-,+)}^{\left(K_{1}\right)} & U_{1}=-1 & U_{2}=-1 & U_{3}=+1, \\
D_{(+,+,+)}^{\left(K_{1}\right)} & U_{1}=+1 & U_{2}=+1 & U_{3}=+1 .
\end{array}
$$

d) Open interval $] \Gamma I_{1}\left[\left(k_{1}=k_{2}<\pi, k_{3}=0\right)\right.$

$$
\text { One gets } T_{1}=T_{2}=\left(\begin{array}{cccc}
c_{1} & -i s_{1} & 0 & 0 \\
-i s_{1} & c_{1} & 0 & 0 \\
0 & 0 & c_{1} & -i s_{1} \\
0 & 0 & -i s_{1} & c_{1}
\end{array}\right) T_{3}=1 \text {. }
$$

The two 2-dimensional unirreps follow:

$$
\begin{array}{llll}
D_{\left(\sigma_{3},-\sigma_{3},-1\right)}^{\left(\Gamma I_{1}\right)} & U_{1}=\sigma_{3} & U_{2}=-\sigma_{3} & U_{3}=-1 \\
D_{\left(-\sigma_{3},-\sigma_{3},-1\right)}^{\left(\Gamma I_{1}\right)} & U_{1}=-\sigma_{3} & U_{2}=-\sigma_{3} & U_{3}=+1 .
\end{array}
$$

e) Open interval $] I_{1} K_{1}\left[\left(k_{1}=k_{3}+\pi, k_{2}=\pi\right)\right.$

$$
\text { One gets } T_{1}=-T_{3}=\left(\begin{array}{cccc}
-c_{3} & 0 & i s_{3} & 0 \\
0 & -c_{3} & 0 & i s_{3} \\
i s_{3} & 0 & -c_{3} & 0 \\
0 & i s_{3} & 0 & -c_{3}
\end{array}\right) T_{2}=-1
$$


and the two 2-dimensional unirreps

$$
\begin{array}{llll}
D_{\left(\sigma_{3},-1,-\sigma_{3}\right)}^{\left(I_{1} K_{1}\right)} & U_{1}=\sigma_{3} & U_{2}=-1 & U_{3}=-\sigma_{3} \\
D_{\left(-\sigma_{3}, 1,-\sigma_{3}\right)}^{\left(I_{1} K_{1}\right)} & U_{1}=-\sigma_{3} & U_{2}=1 & U_{3}=-\sigma_{3}
\end{array}
$$

f) Open interval $] C_{1} K_{1}\left[\left(k_{1}=2 \pi, k_{2}=k_{3}\right)\right.$

$$
\text { One gets } T_{1}=1 \quad T_{2}=T_{3}=\left(\begin{array}{cccc}
c_{3} & 0 & 0 & -i s_{3} \\
0 & c_{3} & -i s_{3} & 0 \\
0 & -i s_{3} & c_{3} & 0 \\
-i s_{3} & 0 & 0 & c_{3}
\end{array}\right) \text {, }
$$

and the two 2-dimensional unirreps

$$
\begin{array}{llll}
D_{\left(1,-\sigma_{3},-\sigma_{3}\right)}^{\left(C_{1} K_{1}\right)} & U_{1}=1 & U_{2}=-\sigma_{3} & U_{3}=-\sigma_{3} \\
D_{\left(-1, \sigma_{3},-\sigma_{3}\right)}^{\left(C_{1} K_{1}\right)} & U_{1}=-1 & U_{2}=\sigma_{3} & U_{3}=-\sigma_{3}
\end{array}
$$

The interesting intervals and points of the boundary of $B_{123}^{-}$are represented on Fig. 4 .

4. The dual of the group. The dual of the group is constructed in two steps. The fact that we are using the Brillouin torus for this construction does not mean that this is always the easiest way for every crystallographic group. We will show, in a forthcoming paper, the difficulties we encounter if we try to construct the dual of a nonsymmorphic group with the aid of the Brillouin torus.

The two steps are the following:

1) We will examine first the boundary of $B_{123}^{-}$is order to derive the topology of its closure denoted $\boldsymbol{B}_{\mathbf{1 2 3}}^{-}$.

2) Then we will consider the union of $\boldsymbol{B}_{\mathbf{1 2 3}}^{-}, \boldsymbol{B}_{\mathbf{2 3 1}}^{-}$and $\boldsymbol{B}_{\mathbf{3 2 1}}^{-}$and we will identify those points of the boundaries which must be identified.

Our only aim is to know the topology of the dual. We are not trying to define a system of coordinates for the dual (we did it for the one-dimensional non-trivial crystallographic group in introducing the parameter $\kappa$ ). That is why we authorize ourselves to perform any continuous deformation of the $B_{i j k^{\prime}}^{-} s$. The first step is described in Fig. 6, the second in Fig. 7.

For the first step it is convenient to give $B_{123}^{-}$the shape of a ball; then its boundary (the one which appears in the Brillouin torus) is spherical. The successive deformations shown in Fig. 6 permit the following identifications: (a) that of the semi-closed triangles $I_{1} J K_{1}$ and $I_{1} L K_{1}$; these two triangles concern identical (fourdimensional) unirreps; the identification is described in the Brillouin torus by a folding around $I_{1} K_{1}$; these triangles are said to be semi- closed because the identification also concerns the open intervals $I_{1} J$ and $I_{1} L_{1}$, the open intervals $K J_{1}$ and $K L_{1}$, and the points $J$ and $L_{1}$; (b) that of the closed intervals $M_{1} C_{1}$ and $J \Gamma$. The final result of the successive deformations, namely $\boldsymbol{B}_{\mathbf{1 2 3}}^{-}$is shown in Fig. 6d. The open intervals $I_{1} K_{1}, K_{1} \Gamma$ and $\Gamma I_{1}$ are double intervals since they describe two distinct sets of two-dimensional representations. For the sake of completude, we have supposed the Brillouin torus to be oriented and this information is taken into account in the successive deformations (see arrows).

The second step consists in sticking the three objects analogous to $\boldsymbol{B}_{\mathbf{1 2 3}}^{-}$, namely $\boldsymbol{B}_{\mathbf{1 2 3}}^{-}$itself, $\boldsymbol{B}_{\mathbf{2 3 1}}^{-}$, and $\boldsymbol{B}_{\mathbf{3 1 2}}^{-}$. To be more precise, we have to identify the points $C_{1}$, 


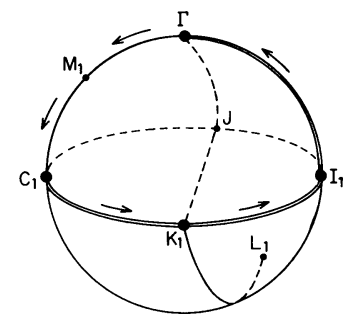

(a)

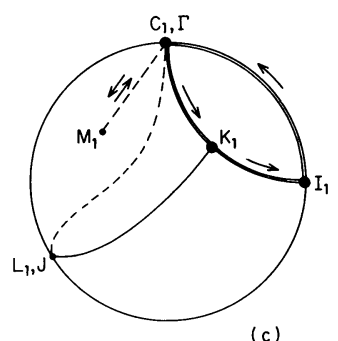

(c)

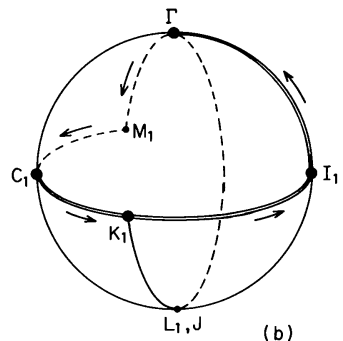

(b)

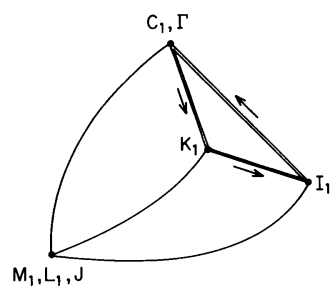

(d)

Fig. 6. The successive deformations of the closure of $B_{123}^{-}$
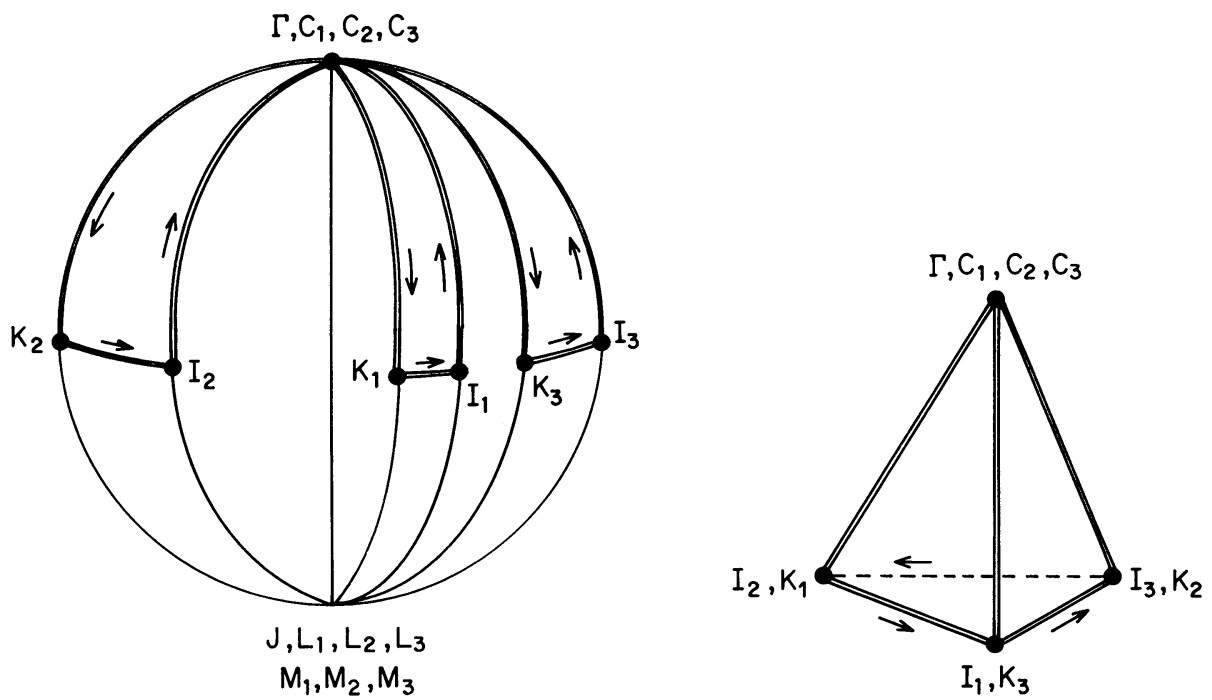

Fig. 7. The construction of the dual of the group F 222

$C_{2}$, and $C_{3}$ with $\Gamma$ and the points $L_{1}, L_{2}, L_{3}, M_{1}, M_{2}, M_{3}$ with $J$, as shown on Fig. 7. Finally we identify the following pairs of faces: $\left(\Gamma I_{1} J \Gamma, \Gamma K_{3} J \Gamma\right),\left(\Gamma I_{2} J \Gamma\right.$, $\left.\Gamma K_{1} J \Gamma\right)$, and $\left(\Gamma I_{3} J \Gamma, \Gamma K_{2} J \Gamma\right)$. The final result is equivalent to a tetrahedron, but a tetrahedron with special features.

1) The union of the open part $V$ of the tetrahedron and of open faces corresponds to the set of all four-dimensional unirreps. 
2) It is non-separated: each open edge is double and each vertex is quadruple.

3) The union of the open edges corresponds to the set of all two-dimensional unirreps. The union of all vertices describe the set of the one-dimensional unirreps. (sixteen in number).

4) This dual space remembers only one thing from the orientation of the Brillouin torus, namely the orientation of the closed contour $K_{1} K_{2} K_{3}$.

5) The topology of the set of lower dimensional unirreps has apparently a very complicated topology which is described in Figs. 8 and 9.

The classification of unirreps is the following one

1. Four dimensional unirreps. We know that the set $V$ is the union of the three parts $B_{123}^{-}, B_{231}^{-}$, and $B_{312}^{-}$. It is a dense subset of the four-dimensional unirreps set. For $B_{123}^{-}$we already know that the unirreps are described by Eqs. (4.7) and (4.8). For $B_{231}^{-}$and $B_{312}^{-}$we have to perform cyclical permutations. It is simple matter to show
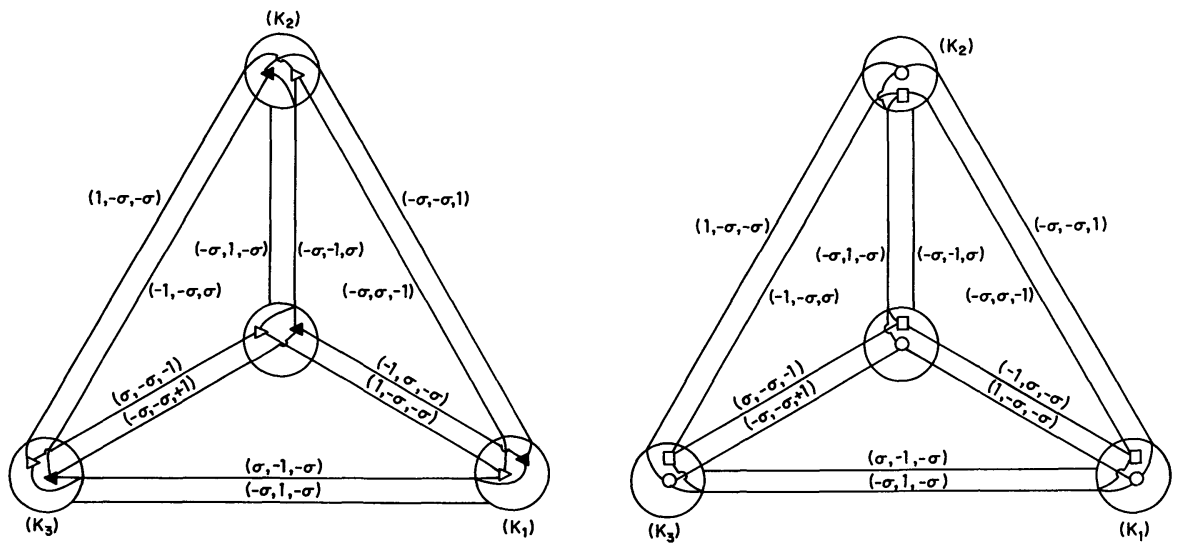

Fig. 8. a The topology of the boundary of the dual of the group $F 222$. Aspects involving the unirreps of type $(+++)$ and $(-+-)$, represented by a circle and a square, respectively. b The topology of the boundary of the dual of the group $F 222$. Aspects involving the unirreps of type $(+--)$ and $(--+)$, represented by a white and a black triangle, respectively

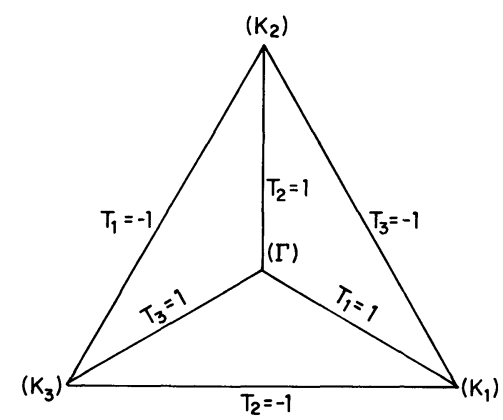

Fig. 9. Translations generators and the boundary of the dual of the group $F 222$ 
that these permutations are described by the following matrices:

$$
\left(\begin{array}{llll}
1 & 0 & 0 & 0 \\
0 & 0 & 1 & 0 \\
0 & 0 & 0 & 1 \\
0 & 1 & 0 & 0
\end{array}\right) \text { and }\left(\begin{array}{llll}
1 & 0 & 0 & 0 \\
0 & 0 & 0 & 1 \\
0 & 1 & 0 & 0 \\
0 & 0 & 1 & 0
\end{array}\right) \text {. }
$$

We must underline the strange fact that they perform the permutations

but

$$
k_{1} \rightarrow k_{2} \rightarrow k_{3} \quad \text { in Eqs. (4.8), }
$$

$$
U_{1} \rightarrow U_{3} \rightarrow U_{2} \text { in Eqs. (4.7). }
$$

2. Unirreps of the six double edges and the four quadruple vertices. Each edge and vertex is characterized by information about the operators $U_{2}$ and at least one of the $T_{i^{\prime}} s$. The unirreps are described in Table 1.

The topology of this part of the dual can be easily understood if we use our notation for the unirreps. We recall that the notation is only based on the way the operators $U_{i}$ 's are represented. Since these operators are always diagonal and since we are only concerned with unirreps of dimension one and two, each $U_{i}$ is represented either by \pm 1 (unirreps of dimension one) or by $\pm \sigma_{3}$, where $\sigma_{3}$ denotes the usual Pauli matrix (for a shorter notation it is simply denoted $\sigma$ ). At each vertex, we have four unirreps, denoted $(+,+,+),(+,-,-),(-,+,-),(-,-,+)$ respectively, where the signs indicate the eigenvalues \pm 1 of the operators $U_{1}, U_{2}, U_{3}$ (in this order). A unirrep of the type $(+,+,+)$ is necessarily in the neighbourhood of a two-dimensional representation of one of the following types $( \pm \sigma, \pm \sigma, 1),(1, \pm \sigma, \pm \sigma),( \pm \sigma, 1, \pm \sigma)$ since they are the only unirreps of dimension two which contain the eigenvalues $(+,+,+)$. Similarly, a unirrep of type $(+,-,-)$ is necessarily in the neighbourhood of a two-dimensional representation of the following types $( \pm \sigma, \pm \sigma,-1),( \pm \sigma,-1, \pm \sigma),(1, \pm \sigma, \pm \sigma)$. It is a simple exercise to show that the dual has eight maximal compact subduals. These can be labelled with the help of the one-dimensional unirreps in $\Gamma, K_{1}, K_{2}, K_{3}$ as follows:

$$
\begin{array}{llll}
a b_{1}:(+++)(+++)(+++)(+++) & c d_{1}:(+++)(+--)(-+-)(--+) \\
a b_{2}:(+--)(+--)(+--)(+--) & c d_{2}:(+--)(+++)(--+)(-+-) \\
a b_{3}:(-+-)(-+-)(-+-)(-+-) & c d_{3}:(-+-)(--+)(+++)(+--) \\
a b_{4}:(--+)(--+)(--+)(--+) & c d_{4}:(--+)(-+-)(+--)(+++)
\end{array}
$$

5. Band representations. The band representation obtained from an arbitrary localized function, that is without any symmetry property ${ }^{8}$ (corresponding to the generic Wyckoff position), has the following decomposition: four times the direct integral on the open part $V$ of the dual (four-dimensional irreps), four times the direct integral on the open faces, twice the direct integral on the open double edges of the dual (two-dimensional irreps) and the direct sum of the sixteen one-dimensional unitary representations associated with the vertices of the dual. It is the regular representation.

It is more interesting to examine the elementary band representations induced by one of the one-dimensional irreducible representations of one of the little groups $D_{2}$ of Wyckoff positions, that is the band representations obtained from symmetric functions. Here we only examine the band representations built from two functions

8 Let us repeat that we have a band representation if the Fourier transform of the function has no zero 
$f^{(a)}$ and $f^{(b)}$ corresponding to Wyckoff positions $(a)$ and (b), respectively. To simplify we only consider the case where these functions are invariant under the little groups $D_{2}$ of the two Wyckoff positions. The other cases (other one-dimensional unirreps of $D_{2}$ ) can be studied in a similar way.

The functions have the following properties:

$$
\begin{aligned}
& f^{(a)}(x, y, z)=f^{(a)}(x,-y,-z)=f^{(a)}(-x, y,-z)=f^{(a)}(-x,-y, z), \\
& f^{(b)}(x, y, z)=f^{(b)}(x,-y, 1-z)=f^{(b)}(-x, y, 1-z)=f^{(b)}(-x,-y, z) .
\end{aligned}
$$

The first function is symmetric with respect to the origin $(0,0,0)$ although the second is symmetric with respect to the point $\left(0,0, \frac{1}{2}\right)$. We may have chosen instead functions with analogous symmetry with respect to equivalent points (representative of the same Wyckoff position).

We are first going to show that the two elementary band representations obtained from these two functions have the same unirreps decomposition. This decomposition concerns the maximal compact subdual denoted $\alpha_{1}$. As we said in our introduction, this property does not mean that the two representations are equivalent.

In order to obtain the unirreps content of the two band representations, we will use the method already used in part 3 of the present paper for the non-trivial onedimensional crystallographic group. We introduce the four following functions:

$$
\begin{aligned}
\psi_{1\left(k_{1}, k_{2}, k_{3}\right)}^{(a, b)}(x, y, z)= & \sum_{n_{1} n_{2} n_{3}} \exp \left[i k_{1} n_{1}+i k_{2} n_{2}+i k_{3} n_{3}\right] \\
& \times f^{(a, b)}\left(x-\frac{n_{2}+n_{3}}{2}, y-\frac{n_{3}+n_{1}}{2}, z-\frac{n_{1}+n_{2}}{2}\right), \\
\psi_{2\left(k_{1}, k_{2}, k_{3}\right)}^{(a, b)}(x, y, z)= & \sum_{n_{1} n_{2} n_{3}} \exp \left[-i k_{1}\left(n_{1}+n_{2}+n_{3}\right)+i k_{2} n_{3}+i k_{3} n_{2}\right] \\
& \times f^{(a, b)}\left(x-\frac{n_{2}+n_{3}}{2}, y-\frac{n_{3}+n_{1}}{2}, z-\frac{n_{1}+n_{2}}{2}\right), \\
\psi_{3\left(k_{1}, k_{2}, k_{3}\right)}^{(a, b, y}(x)= & \sum_{n_{1} n_{2} n_{3}} \exp \left[i k_{1} n_{3}-i k_{2}\left(n_{1}+n_{2}+n_{3}\right)+i k_{3} n_{1}\right] \\
& \times f^{(a, b)}\left(x-\frac{n_{2}+n_{3}}{2}, y-\frac{n_{3}+n_{1}}{2}, z-\frac{n_{1}+n_{2}}{2}\right), \\
\psi_{4\left(k_{1}, k_{2}, k_{3}\right)}^{(a, b, y, z)=} & \sum_{n_{1} n_{2} n_{3}} \exp \left[i k_{1} n_{2}-i k_{2} n_{1}-i k_{3}\left(n_{1}+n_{2}+n_{3}\right)\right] \\
& \times f^{(a, b)}\left(x-\frac{n_{2}+n_{3}}{2}, y-\frac{n_{3}+n_{1}}{2}, z-\frac{n_{1}+n_{2}}{2}\right) .
\end{aligned}
$$

It is easy to check that if the generators act on a function as follows:

$$
\begin{aligned}
& t_{1} f(x, y, z)=f\left(x, y-\frac{1}{2}, z-\frac{1}{2}\right), \\
& t_{2} f(x, y, z)=f\left(x-\frac{1}{2}, y, z-\frac{1}{2}\right), \\
& t_{3} f(x, y, z)=f\left(x-\frac{1}{2}, y-\frac{1}{2}, z\right), \\
& u_{1} f(x, y, z)=f(x,-y,-z), \\
& u_{2} f(x, y, z)=f(-x, y,-z), \\
& u_{3} f(x, y, z)=f(-x,-y, z),
\end{aligned}
$$


the generators of translations act as the matrices of Eq.(4.4) either on the space spanned by the four functions $\psi_{i\left(k_{1}, k_{2}, k_{3}\right)}^{(a)}(x, y, z)$ or on the space spanned by the four functions $\psi_{i\left(k_{1}, k_{2}, k_{3}\right)}^{(b)}(x, y, z)$. However, the generators of $\pi$-rotations act differently on these two spaces. We get

$$
\begin{aligned}
& U_{1}^{(a)}=\left(\begin{array}{llll}
0 & 1 & 0 & 0 \\
1 & 0 & 0 & 0 \\
0 & 0 & 0 & 1 \\
0 & 0 & 1 & 0
\end{array}\right) \quad U_{2}^{(a)}=\left(\begin{array}{cccc}
0 & 0 & 1 & 0 \\
0 & 0 & 0 & 1 \\
1 & 0 & 0 & 0 \\
0 & 1 & 0 & 0
\end{array}\right) \quad U_{3}^{(a)}=\left(\begin{array}{cccc}
0 & 0 & 0 & 1 \\
0 & 0 & 1 & 0 \\
0 & 1 & 0 & 0 \\
1 & 0 & 0 & 0
\end{array}\right) \\
& U_{1}^{(b)}=\left(\begin{array}{cccc}
0 & \alpha & 0 & 0 \\
\alpha^{*} & 0 & 0 & 0 \\
0 & 0 & 0 & \alpha^{*} \\
0 & 0 & \alpha & 0
\end{array}\right) U_{2}^{(b)}=\left(\begin{array}{cccc}
0 & 0 & \alpha & 0 \\
0 & 0 & 0 & \alpha^{*} \\
\alpha^{*} & 0 & 0 & 0 \\
0 & \alpha & 0 & 0
\end{array}\right) \quad U_{3}^{(b)}=\left(\begin{array}{cccc}
0 & 0 & 0 & 1 \\
0 & 0 & 1 & 0 \\
0 & 1 & 0 & 0 \\
1 & 0 & 0 & 0
\end{array}\right),
\end{aligned}
$$

where $\alpha$ is the phase factor $\exp \left[-i\left(k_{1}+k_{2}-k_{3}\right)\right]$ and $\alpha^{*}$ its complex conjugate.

It is a simple matter to check that these four-dimensional "subrepresentations" are equivalent ${ }^{9}$. For an arbitrary triple $\left(k_{1}, k_{2}, k_{3}\right)$ the matrices obtained can be intertwined by the matrix

$$
A\left(k_{1}, k_{2}, k_{3}\right)=\left(\begin{array}{cccc}
\alpha & 0 & 0 & 0 \\
0 & 1 & 0 & 0 \\
0 & 0 & 1 & 0 \\
0 & 0 & 0 & \alpha
\end{array}\right),
$$

that is $A U_{1}^{(a)}=U_{1}^{(b)} A$. This proves that the two band representations have the same contents in unirreps of dimension four.

It is easy to go to the lower dimensional part of the dual and check that the two band representations have also the same contents in two- and in one-dimensional unirreps. For instance, if we suppose $k_{1}>k_{2}>k_{3}$, one sees on the line $\Gamma I_{1}$ (equivalent to $\Gamma K_{3}$ ) that the functions $\psi_{1}$ and $\psi_{4}$ are equal and so are the functions $\psi_{2}$ and $\psi_{3}$, whatever is the Wyckoff position chosen. By use of the matrix transformation $M$ of Eq. (4.6), one readily sees that we only get the irreps $(-\sigma,-\sigma, 1)$. In looking similarly at the edges $I_{1} K_{1}$ (equivalent to $K_{3} K_{1}$ ) and $C_{1} K_{1}$ (equivalent to $\Gamma K_{1}$ ), it can be shown that in both cases, we get the irreps $(-\sigma, 1,-\sigma),(1,-\sigma,-\sigma)$. We can do the same for the other edges and go to the vertices. We readily arrive at the maximal compact subdual $a b_{1}$ or $(+++)(+++)(+++)(+++)$.

We are left with the problem of showing why these representations are not equivalent although they have the same unirreps content. To be equivalent means that the two representations could be intertwined by a unitary operator $A$. Such an operator has a decomposition on $a b_{1}$,

$$
A=\int_{a b_{1}} A(\kappa) d \kappa,
$$

where $A(\kappa)$ is a unitary matrix of dimension 4,2 or 1 , depending on the point $\kappa$. The existence of such a matrix does not prove that $A$ is a unitary operator. In fact Eq.(4.9) provides us with such a unitary matrix $A(\kappa)$. This matrix is labelled by the phase factor $\alpha$. This defines a section of a $U(1)$ bundle of base $a b_{1}$.

\footnotetext{
9 They are not strictly speaking subrepresentations since these four dimensional spaces are not Hilbert subspaces
} 
If $A$ is unitary, it is diagonisable and, in multiplying the basis vectors of the two Hilbert spaces by suitable phase factors, it can be mapped continuously on the unit infinite matrix. This means that the section defined by $A(\kappa)$ would be trivial. But this is not the case. One can easily check that $A(\kappa)$ takes the value 1 on the four open edges $K_{1} K_{2}, K_{2} K_{3}, \Gamma K_{1}, \Gamma K_{3}$ and the four vertices, since on this part the phase factor $\alpha$ takes the value 1 . The problem is that on the two other edges $K_{1} K_{3}$ and $\Gamma K_{2}$, the phase factor varies from 0 to $2 \pi$. The difference between the intertwining matrix $A$ and the unit matrix is similar to the one which exists between a regular band and a Møbius band.

Table 1

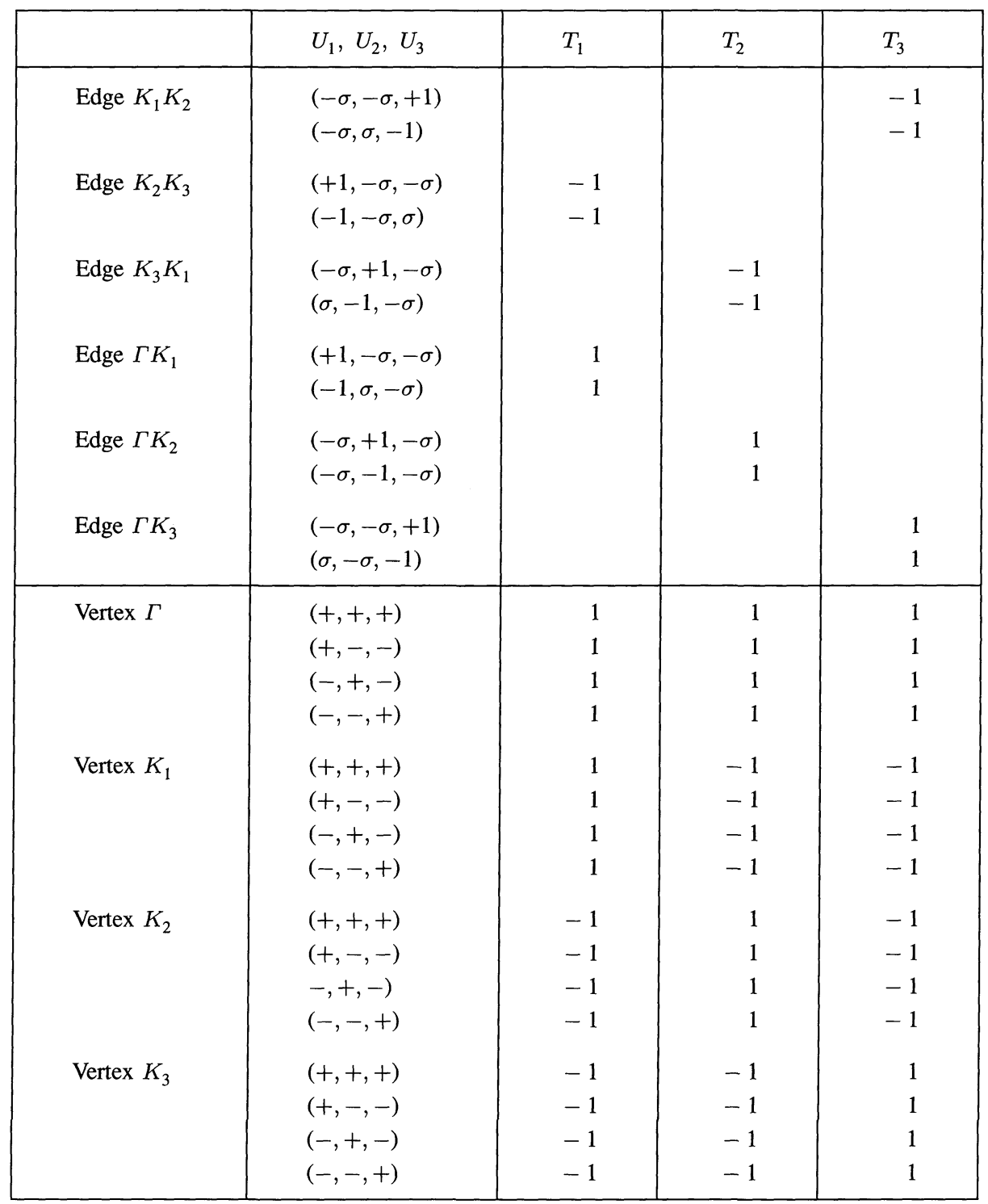




\section{Conclusion}

We have seen that the dual of the symmorphic group $F 222$ has eight maximal compact subduals. According to results of references [9] and [12], one can say the two following things:

1) There exist sixteen elementary band representations; each of them is induced by one of the four one-dimensional unirreps of a stability group associated with one of the four Wyckoff positions $a, b, c, d$.

2) The content of an induced representation associated with Wyckoff position $a$ or $b$ (resp. $c$ or $d$ ) corresponds to a maximal compact subdual of type $a b$ (resp. $c d$ ).

The dual provides us with two types of information about band representations:

1) It permits to visualize a group theoretical result analogous to the one we mentioned for the one-dimensional non-trivial space group: the content of the regular representation is the "sum" of the contents of either the max. comp. subd. of type $a b$ or of the max. comp. subd. of type $c d$.

2) Since every max. comp. subd. has a non-trivial circle bundle, this is an indication that distinct elementary band representations may have identical decomposition. A contrario, it is clear that the four max. comp. subd. of the non-trivial one-dimensional space group only have trivial bundles. That is why we can say that distinct elementary band representations have distinct contents.

The dual also gives indications about quasi-band representations. To clarify this point, let us go back to the two elementary band representations of the group $F 222$ we have studied. We know that if we restrict the Hilbert space in discarding the content associated with the edges $K_{1} K_{3}$ and $\Gamma K_{2}$, we can construct an intertwining operator. It seems to follow that if we choose as a starting function a function for which the Fourier transform contains as a factor the function $\sin \left(p_{1}-p_{3}\right)$, instead of band representations we would get equivalent quasi-band representations.

We have to prove that our bundle section argument is equivalent to the Berry phase argument found by Michel and Zak [7]. These authors perform an integration on the Brillouin zone. It seems necessary to understand how this is related with an integration on the tetrahedron. One point is satisfactory: both arguments correspond to a relative phase.

We must insist on the fact that all these points have no room in the case of Bornvon Karman groups since being finite groups their duals are finite. For them, two band representations which have the same contents are always equivalent.

It is clear that we did not explore in the present article all kinds of facts which can be discovered in band and quasi-band representations of the crystallographic groups. In particular, we were not involved in the difficulties which could rise in the case of multiple branches band representations. We intend to discuss this case in a forthcoming paper.

To conclude, we mention a slightly weaker conjecture.

Conjecture 3. Every elementary band representation is multiplicity free, that is every decomposition into two subrepresentations implies that these two subrepresentations are disjoint.

Acknowledgments. The author is grateful to Joshua Zak for interesting discussions and for his strong interest in this work. He also thanks all members of the Physics Department of the Technion for their warm hospitality. Thanks are also due to Louis Michel for his critical reading of the manuscript and his encouragements. 


\section{References}

1. Hahn, Th. (Editor),: International tables for crystallography, Dordrecht: Reidel, 1987

2. des Cloizeaux, J.: Phys. Rev. 129, 554 (1963)

3. des Cloizeaux, J.: Phys. Rev. 135A, 698 (1964)

4. Zak, J.: Phys. Rev. Lett. 45, 1025 (1980)

5. Zak, J.: Phys. Rev. B25, 1344 (1982)

6. Zak, J.: Phys. Rev. B26, 3010 (1982)

7. Michel, L., Zak, J.: Europhys. Lett. 18, 239 (1992)

8. Bacry, H., Michel, L., Zak, J: Phys. Rev. Lett. 61, 1005 (1988)

9. Bacry, H., Michel, L., Zak, J.: In Lect. Notes in Phys. 313, 291, Berlin-Heidelberg-New York: Springer-Verlag, 1988

10. Zak, J.: Phys. Rev. Lett. 62, 2747 (1989)

11. Zak, J.: In Lect. Notes in Phys. 382, 581 (1990)

12. Bacry, H., Michel, L., Zak, J.: Band representations of space groups: concepts and classification. (to be submitted)

13. Dixmier, J.: Les $C^{*}$-algèbres et leurs représentations (Proposition 3.1.8), Paris: Gauthier-Villars, 1964

14. Mackey, G.W.: The theory of unitary group representations. Chicago: The University of Chicago Press 1976

15. Bacry, H.: How a crystallographic group acts on space. Contribution to the International Colloquium in honor of Louis Michel, Lyon, June 1991

Communicated by A. Connes 